\title{
A Method of Summing Infinite Series
}

\author{
Samuel Lubkin
}

\begin{abstract}
This paper describes a new method of obtaining an equivalent series from a given infinite convergent or divergent series. In many cases the new series is more convenient for summing than the original and, moreover, the same method may usually be repeated indefinitely to obtain an entire sequence of series each equivalent to the original and each better than its predecessor in summing properties. The new method differs from most summing methods heretofore employed in that terms of the transformed series are not linear functions of terms of the original series. The paper includes proofs of theorems indicating the scope of the new method and comparisons of results with various other methods for many specific examples.
\end{abstract}

\section{General}

Infinite series are not always amenable to convenient summing by usual methods. Even when convergent, a series may converge too slowly to permit ready evaluation or may consist of terms which, individually, require so much labor in evaluation as to make determination of sufficient terms for summing even a moderately rapidly convergent series quite difficult. If the series is asymptotic, the magnitude of the smallest term seems to set a lower bound on the accuracy of evaluation. For other types of divergent series, a convenient summing method may not be available. This paper presents a method of transforming series that may be of value in all these cases. To the best of the author's knowledge, the method is original with him, and has not been previously published, although the author has used it since $1937 .^{1}$

Essentially, the method consists of approximating the series after the first $m$ terms by a geometric series whose first term is the $(m+1)$ th term of the original series and whose ratio is the ratio of the $(m+2) \mathrm{d}$ term to the $(m+1)$ th. These approximating sums for successive values of $m$ may be considered to be successive partial sums of a new series that has the same sum as the old but has, frequently, superior summing properties. The process may, in many cases, be repeated again on the new series to get a third series with still better properties. An indefinite number of repetitions may be possible, as will be shown in examples given later.

This method differs from summing methods usually employed in that the terms of the transformed series are not linear functions of the terms of the original series. It should also be pointed out that various modifications of the method are possible such as use of other types of approximating series instead of the simple geometric series, ${ }^{2}$ but loss of simplicity is

\footnotetext{
1 since the turst draft of this paper, the author has learned of a talk presented by D. Shanks at the Naval Ordnance Laboratory, White Oaks, Md. entitled "Mathe. a series as the sum of corresponding terms of one or more geometric series. When a single geometric series is considered, his procedure reduces to that discussed in this paper. More complicated, but generally more effective, transformations this paper. More complicated, but generally more effective, transformations
result from the use of more than one component geometric series. Finally, he has found it possible to use an infinite number of component series, in which case the sum can be represented as a ratio of two determinants of infinite order, which generally converge with considerable rapidity. The author also understands that Otto Szasz has had, for sometime past, a paper under preparation which embodies similar material.

2 Such as the sum of several geometric series as taken by Shanks in the talk referred to in the previous footnote.
}

likely to offset any gain resulting from use of better fitting approximations. ${ }^{3}$

Summarizing, this paper discusses the transformation of a given infinite series:

$$
S=a_{0}+a_{1}+a_{2}+a_{3}+\ldots+a_{n}+a_{n+1}+\ldots
$$

into a new series:

$$
T=b_{0}+b_{1}+b_{2}+b_{3}+\ldots+b_{n}+b_{n+1}+\ldots .
$$

If we define the partial sums by

and

$$
S_{n}=a_{0}+a_{1}+a_{2}+\ldots+a_{n}
$$

$$
T_{n}=b_{0}+b_{1}+b_{2}+\ldots+b_{n},
$$

the $T$ series is defined by the relations

$$
\left.\begin{array}{l}
T_{0}=a_{0} /\left(1-R_{1}\right) ; \\
T_{n}=S_{n-1}+\frac{a_{n}}{1-R_{n+1}}, n>0
\end{array}\right\}
$$

where

$$
R_{n}=a_{n} / a_{n \oplus 1},
$$

defined only for $n>0$.

This relation can also be stated, by minor manipulation, as:

$$
T_{n}=S_{n}+\frac{a_{n+1}}{1-R_{n+1}}
$$

or as

$$
T_{n}=\frac{S_{n}^{2}-S_{n-1} S_{n+1}}{2 S_{n}-S_{n-1}-S_{n+1}}, n>0 ; T_{0}=\frac{S_{0}^{2}}{2 S_{0}-S_{1}},
$$

From these relations, we readily obtain:

$$
\left.\begin{array}{l}
b_{0}=\frac{a_{0}}{1-R_{1}} ; \\
b_{n}=a_{n}\left(\frac{1}{1-R_{n+1}}-\frac{1}{1-R_{n}}\right), n>0
\end{array}\right\}
$$

for the terms of the new series.

Examination of these relations shows that a finite

3 See, however, transformation $W$ below and theorems on its properties. 
alteration (modification or elimination of a finite number of terms) in the original series results oply in a finite alteration in the transformed series. We also note that the conditions $R_{n}=1$ or $a_{n}=0$ require special consideration. In the former case, we get $T_{n-1}=\infty$ to correspond. If this occurs for a finite number of values of $n$, we may a void the difficulty by a suitable finite alteration (such as omission of the first $N$ terms where $N$ exceeds any $n$ for which $R_{n}=1$ ) in the original series. If it occurs for an infinite set of values of $n$, the transformation has little value unless the original series can be rearranged, terms combined, or split into subseries to avord this difficulty. If $a_{n}=0$, we may take $b_{n}=0$ and $T_{n}=S_{n-1}=S_{n}$ and $b_{n+1}=a_{n+1} /\left(1-R_{n+2}\right)$ if $a_{n+1} \neq 0$ but $a_{n}=0$; as a set of relations consistent with those given for $a_{n} \neq 0$. However, $T_{n}=S_{n}$ at those values of $n$ for which $a_{n}=0$ implies that the transformation is no better than can be achieved by adding terms of the original series between successive zeros. Example 3 below is an illustration. The theorems given, however, are valid without specific limitation if we use the relations suggested when $a_{n}=0$ since their hypotheses rule out $R_{n}=1$ at an infinite set of values of $n$ automatically or the results follow even if this is the case.

For convenience in later discussion, we add the following definitions:

$$
R=\lim _{n \rightarrow \infty} R_{n} .
$$

$Q_{n}=n\left(1-R_{n}\right)$, defined only for $n>0$.

$$
Q=\lim _{n \rightarrow \infty} Q_{n} .
$$

$P_{0}=b_{0} / a_{0}=1 /\left(1-R_{1}\right)$.

$\left.P_{n}=\frac{b_{n}}{a_{n}}=\frac{1}{1-R_{n+1}}-\frac{1}{1-R_{n}}=\frac{R_{n+1}-R_{n}}{\left(1-R_{n+1}\right)\left(1-R_{n}\right)}, n>0.\right\}$

We shall also make use of another transformation defined by the relatious:

$$
\begin{gathered}
W=w_{0}+w_{1}+w_{2}+\ldots+w_{n}+w_{n+1}+\ldots, \\
W_{n}=w_{0}+w_{1}+w_{2}+\ldots+w_{n}, \\
=\frac{T_{n}-P_{n} S_{n}}{1-P_{n}} .
\end{gathered}
$$

From these, we readily obtain:

$$
\begin{aligned}
W_{n} & =S_{n-1}+\frac{a_{n}\left(1-R_{n+1}\right)}{1-2 R_{n+1}+R_{n} R_{n+1}}, n>0, \\
& =S_{n}+\frac{a_{n+1}\left(1-R_{n}\right)}{1-2 R_{n+1}+R_{n} R_{n+1}}, n>0 .
\end{aligned}
$$

$$
w_{0}=0 ; w_{1}=a_{1}\left[\frac{1-R_{2}}{1-2 R_{2}+R_{1} R_{2}}+\frac{1}{R_{1}}\right] \text {; }
$$$$
\left.w_{n}=a_{n}\left[\frac{1-R_{n+1}}{1-2 R_{n+1}+R_{n} R_{n+1}}-\frac{1-R_{n-1}}{1-2 R_{n}+R_{n} R_{n-1}}\right], n>1 .\right\}
$$

The $W$ transformation is similar to the $T$ transformation in that a finite alteration in $S$ causes only a finite alteration in $W$. Such an alteration may be used to avoid consideration of a finite number of terms for which either $a_{n}=0$ or $1-2 R_{n}+R_{n} R_{n-1}=0$. The transformation is obviously not of value if an infinite number of terms have either property. As for the $T$ transformation, we shall take $w_{n}=0$ whenever $a_{n}=0$. The relation $1-2 R_{n}+R_{n} R_{n-1} \neq 0$ will be assured, except for a finite number of terms at most, by the conditions of the theorems concerned.

In accord with usual terminology, we shall say that a series $C=\sum_{0}^{\infty} c_{n}$ with partial sums $C_{n}=\sum_{0}^{n} c_{m}$ is more rapidly convergent than $S$ if both $S$ and $C$ converge and $\left(C-C_{n}\right) /\left(S-S_{n}\right)$, the ratio of the corresponding remainders, tends to zero as $n$ tends to infinity; is of the same order of rapidity of convergence if both series converge and $\mid\left(C-C_{n}\right) /$ $\left(S-S_{n}\right) \mid$ remains in value between two finite positive constants for all sufficiently large $n$; and is no less rapidly convergent than $\dot{S}$ if both series converge and the ratio of corresponding remainders is bounded as $n \rightarrow \infty$.

The remainder of this paper makes use of the common definitions and theorems, generally without specific acknowledgment. Where such material is believed less well known, reference will be made by footnote to a source. For convenience, wherever possible, such reference will be made to the "Theory and application of infinite series" by Konrad Knopp, English Edition (1928) and will consist merely of the name Knopp followed by the page number in this text.

No extensive bibliography is included in this paper. The reader will find appropriate references to other sources in the book by Knopp mentioned above and in a report by $R$. D. Carmichael in the Bulletin of the American Mathematical Society, 25, pp. 97 to 131 (1918) on the subject "General aspects of the theory of summable series". An extensive bibliography on similar material will also be found in "Studies on divergent series and summability" by W. B. Ford (Macmillan, New York, N. Y. (1916)). Since the first draft of this paper, a book by G. H. Hardy, titled "Divergent series", has been published by the Oxford University Press. The following are several papers dealing with methods of obtaining sums of slowly convergent series that appeared more recently:

J. A. Shohat, On a certain transformation of infinite series, Am. Math. Monthly 40, 226 to 229 (1933).

W. G. Bickley and J. C. P. Miller, The numerical summation of slowly convergent series of positive terms, Phil. Mag. [7], 22, 754 to 767 (1936).

J. W. Bradshaw, Modified continued fractions for certain series, Am. Math. Monthly 45, 352 to 362 (1938).

J. W. Bradshaw, Modified series, Am. Math. Monthly 46, 486 to 492 (1939).

O. Szasz, Summation of slowly convergent series, presented before Am. Math. Soc. June 19, 1948, and in J. Math. Phys. 28, 272 (1950). 


\section{Original Series Convergent ${ }^{4}$}

In this section, the transformation $T$ (and, in certain cases, $W$ ) will be considered in connection with an original series $S$, which is convergent. Theorems will be stated and proved that give some of the conditions under which the transformation leads to a convergent series; beginning with one that states that, in this case, the derived series has the same sum as the original. Since, in connection with a convergent series, the transformation is chiefly intended to permit more convenient numerical evaluation of the sum, we shall be mainly concerned with the relative rapidity of convergence of the derived and original series.

It is convenient to have criteria on rapidity of convergence that involve relative magnitudes of individual terms of the series considered rather than of remainders. A group of lemmas on this subject thus precedes the formal part of the discussion. The section closes with a number of numerical examples that show some of the types of behavior that may be encountered.

Lemma 1. If $S$ is convergent with $R_{n}>0$ for $n$ sufficiently large, then the series $C=\sum_{0}^{\infty} c_{n}$ converges with the same order of rapidity as $S$ if there exist constants $A, B$, and $N$ such that $A B>0$ and $A>\mathrm{c}_{n} /$ $a_{n}>B$ for all $n>N$; no less rapidly than $S$ if $c_{n} / a_{n}$ is bounded as $n \rightarrow \infty$; and more rapidly than $S$ if $c_{n} / a_{n} \rightarrow 0$ as $n \rightarrow \infty$.

Proof: We restrict ourselves throughout to $n$ sufficiently large without specific statement to that effect. Since $R_{n}>0$, all $a_{n}$ are of the same sign, which we may assume to be positive. Then, since $\left|c_{n} / a_{n}\right|$ is bounded for all cases considered, $C$ converges. ${ }^{5}$ If $A>c_{n} / a_{n}>B$, we have $A a_{n}>c_{n}>B a_{n}$ and $A \sum_{n}^{\infty} a_{m}>$ $\sum_{n}^{\infty} c_{m}>B \sum_{n}^{\infty} a_{m}$ so that $A>\sum_{n}^{\infty} c_{m} / \sum_{n}^{\infty} a_{m}>B$. If $A B>0$, then $A$ and $B$ are of like sign and $\left|\sum_{n}^{\infty} c_{m}\right| \sum_{n}^{\infty} a_{m} \mid$ has a value between $|A|$ and $|B|$ and hence $C$ converges with the same order of rapidity as $S$. If $c_{n} / a_{n}$ is bounded, $A$ and $B$ exist but may be of opposite sign. In this case, $\left|\sum_{n}^{\infty} c_{m}\right| \sum_{n}^{\infty} a_{m} \mid<D$ where $D$ is the larger of $|A|$ and $|B|$ and hence $C$ converges no less rapidly than $S$. If $c_{n} / a_{n} \rightarrow 0$, then, for any $\epsilon>0$, we have $\epsilon>c_{n} / a_{n}>-\epsilon$ for $n$ sufficiently large. Hence $\left|\sum_{n}^{\infty} c_{m} / \sum_{n}^{\infty} a_{m}\right|<\epsilon \rightarrow 0$ as $n \rightarrow \infty$ so that $C$ converges more rapidly than $S$.

Lemma 2. If $S$ is convergent with $R_{n} R_{n-1}\left(1+R_{n+1}\right)$ $\times\left(1+R_{n-1}\right)>0$ for $n$ sufficiently large, and the series $C=\sum_{0}^{\infty} c_{n}$ has terms such that $c_{n} \rightarrow 0$ as $n \rightarrow \infty$, then $C$ converges with the same order of rapidity as $S$ if there exist constants $A, B$, and $N$ such that $A B>0$ and

4 Unless otherwise stated, nomenclature is that given in section 1 . We also use the same symbol to represent a series and its sum.

5 Knopp, p. 137.
$A>\left(c_{n}+c_{n+1}\right) /\left(a_{n}+a_{n+1}\right)>B$ for all $n>N$; no less rapidly than $S$ if $\left(c_{n}+c_{n+1}\right) /\left(a_{n}+a_{n+1}\right)$ is bounded as $n \rightarrow \infty$; and with greater rapidity than $S$ if $\left(c_{n}+c_{n+1}\right) /$ $\left(a_{n}+a_{n+1}\right) \rightarrow 0$ as $n \rightarrow \infty$.

Proof: We assume $n$ sufficiently large. Then

$$
\begin{aligned}
\frac{a_{n}+a_{n+1}}{a_{n-2}+a_{n-1}} & =\frac{a_{n}\left(1+R_{n+1}\right)}{a_{n-2}\left(1+R_{n-1}\right)} \\
& =\frac{R_{n} R_{n-1}\left(1+R_{n+1}\right)\left(1+R_{n-1}\right)}{\left(1+R_{n-1}\right)^{2}}>0 .
\end{aligned}
$$

The series $S^{(1)}=\sum_{0}^{\infty}\left(a_{2 n}+a_{2 n+1}\right) \quad$ and $\quad S^{(2)}=a_{0}+$ $\sum_{0}^{\infty}\left(a_{2 n-1}+a_{2 n}\right)$ both converge and have the sum $S$ since $S_{n}^{(1)}=S_{2 n+1} \rightarrow S$ and $S_{n}^{(2)}=S_{2 n} \rightarrow S$. By Lemma 1 , the series $C^{(1)}=\sum_{0}^{\infty}\left(c_{2 n}+c_{2 n+1}\right)$ converges with the indicated rate for each condition as compared to series $S^{(1)}$ and the same holds for the series $C^{(2)}=c_{0}+$ $\sum_{1}^{\infty}\left(c_{2 n-1}+c_{2 n}\right)$ as compared to $S^{(2)}$. Now $C_{n}^{(1)}=C_{n}^{(2)}+$ $c_{2 n+1} \sim C_{n}^{(2)} \rightarrow C^{(2)} \quad$ since $c_{n} \rightarrow 0$. Hence $C^{(1)}=C^{(2)}$. Furthermore, $\quad C_{2 n}=C_{n}^{(2)} \rightarrow C^{(2)}$ and $C_{2 n+1}=C_{n}^{(1)} \rightarrow$ $C^{(1)}=C^{(2)}$. Hence $C$ converges and $C=C^{(2)}=C^{(1)}$. We now have

and

$$
\frac{C-C_{2 n}}{S-S_{2 n}}=\frac{C^{(2)}-C_{n}^{(2)}}{S^{(2)}-S_{n}^{(2)}},
$$

$$
\frac{C-C_{2 n+1}}{S-S_{2 n+1}}=\frac{C^{(1)}-C_{n}^{(1)}}{S^{(1)}-S_{n}^{(1)}},
$$

hence proving the lemma in view of the known rates of convergence of $C^{(2)}$ as compared to $S^{(2)}$ and $C^{(1)}$ as compared to $S^{(1)}$.

Lemma 3. If $S$ is convergent with $R_{n} R_{n-1}\left(1+R_{n+1}\right)$ $\left(1+R_{n+1}\right)>0$ and $\left|1+R_{n}\right|>K>0$ for $n$ sufficiently large, then the series $C=\sum_{0}^{\infty} c_{n}$ converges no less rapidly than $S$ if $c_{n} / a_{n}$ is bounded as $n \rightarrow \infty$ and more rapidly than $S$ if $c_{n} / a_{n} \rightarrow 0$ as $n \rightarrow \infty$.

Proof: Since $S$ converges, $a_{n} \rightarrow 0$. Hence, for either condition, $c_{n} \rightarrow 0$. Also, for $n$ sufficiently large,

$$
\begin{aligned}
\left|\frac{c_{n}+c_{n+1}}{a_{n}+a_{n+1}}\right| & =\left|\frac{c_{n}}{a_{n}\left(1+R_{n+1}\right)}+\frac{c_{n+1}}{a_{n+1}}-\frac{c_{n+1}}{a_{n+1}\left(1+R_{n+1}\right)}\right| \\
& <\frac{1}{K}\left|\frac{c_{n}}{a_{n}}\right|+\left|\frac{c_{n+1}}{a_{n+1}}\right|+\frac{1}{K}\left|\frac{c_{n+1}}{a_{n+1}}\right|
\end{aligned}
$$

and is thus bounded (or $\rightarrow 0$ ) when $c_{n} / a_{n}$ is bounded $($ or $\rightarrow 0)$. We may now apply Lemma 2 .

Remark. The condition $R_{n} R_{n-1}\left(1+R_{n+1}\right)\left(1+R_{n-1}\right)>0$ is satisfied if $R_{n}>0$ for all $n$ or if $R_{n}<0$ and $\left(1+R_{n+1}\right)\left(1+R_{n-1}\right)>0$ for all $n$. A special case of the latter is $0>R_{n}>-1$.

Theorem 1. If both $S$ and $T$ converge, they have the same sum. 
Proof: Suppose $T \neq S$. Then, from (7),

$$
a_{n} /\left(1-R_{n}\right)=T_{n-1}-S_{n-1} \rightarrow T-S \neq 0 .
$$

Since $S$ converges, $a_{n} \rightarrow 0$. Hence, from the above, $1-R_{n} \rightarrow 0$ or $R_{n} \rightarrow 1$. Therefore, for $n$ sufficiently large, $R_{n}>0$ and all $a_{n}$ are of like sign. In view of the above, this requires that $1-R_{n}$ be of constant sign for $n$ sufficiently large. Hence, either $R_{n}>1$ for all sufficiently large $n$ or $R_{n}<1$ for all sufficiently large $n$. The former is impossible since $a_{n} \rightarrow 0$. The latter, coupled with the constancy of sign of the $a_{n}$, states that, for $n$ sufficiently large, either $S$ or $-S$ consists of positive monotonically decreasing terms. Convergence of $S$ thus requires that $n a_{n} \rightarrow 0 .{ }^{6}$ However,

$$
\frac{n a_{n}}{n\left(1-R_{n}\right)}=\frac{a_{n}}{1-R_{n}} \rightarrow T-S \neq 0
$$

Hence we must have $n\left(1-R_{n}\right) \rightarrow 0$. This is not possible since $S$ converges. ${ }^{7}$ Therefore our supposition cannot be true, and $T=S$.

Theorem 2. If $S$ is convergent and $\left|1-R_{n}\right|>K_{1}>0$ for sufficiently large $n$, then $T$ converges. If, in addition $\left|1+R_{n}\right|>K_{2}>0$ and $R_{n} R_{n-1}\left(1+R_{n+1}\right)\left(1+R_{n-1}\right)>0$ for $n$ sufficiently large, 'I' converges no less rapidly than $S$. If furthermore, $R_{n+1}-R_{n} \rightarrow 0$ as $n \rightarrow \infty$, then $T$ converges with greater rapidity than $S$.

Proof: Since $S$ converges, $\quad a_{n} \rightarrow 0$. Hence $\left|a_{n} /\left(1-R_{n}\right)\right|<\left|a_{n}\right| / K_{1} \rightarrow 0$ and, from (7), $T_{n} \sim S_{n} \rightarrow S$. Also, from (13)

$$
\left|P_{n}\right|<\frac{1}{\left|1-R_{n+1}\right|}+\frac{1}{\left|1-R_{n}\right|}<\frac{2}{K_{1}},
$$

and thus remains bounded while, if $R_{n+1}-R_{n} \rightarrow 0$,

$$
\left|P_{n}\right|=\frac{\left|R_{n+1}-R_{n}\right|}{\left|1-R_{n+1}\right|\left|1-R_{n}\right|}<\frac{\left|R_{n+1}-R_{n}\right|}{K_{1}^{2}} \rightarrow 0 .
$$

Application of Lemma 3 now completes the proof.

Theorem 3. If $S$ converges and $R$ exists $\neq 0,-1$, or 1 , then $T$ converges with greater rapidity than $S$.

Proof: Since $S$ converges, $|R| \leq 1$. But $|R| \neq 1$ by hypothesis. Hence, $|R|<1$. Now $R_{n} \rightarrow R$. Hence, for $n$ sufficiently large, $1 \pm R_{n}>(1-|R|) / 2>0$. If $R>0$ (or $<0)$, then $R_{n}>0$ (or $\left.<0\right)$ for $n$ sufficiently large. In either case, $R_{n} R_{n-1}>0$ for $n$ sufficiently large. We also note that $R_{n} \rightarrow R$ requires $R_{n+1}-R_{n} \rightarrow 0$, and we therefore satisfy all the conditions of 'Theorem 2.

Theorem 4. If $S$ converges, $R=0$, and $R_{n}$ is of constant sign for $n$ sufficiently large, then $T$ converges with greater rapidity than $S$.

Proof: Since $R_{n} \rightarrow 0$, wa have $1 \pm R_{n}>\frac{1}{2}>0$ for $n$ sufficiently large. Also $R_{n} R_{n-1}>0$, since $R_{n}$ is of constant sign, for $n$ sufficiently large. We now apply Theorem 2, noting that $R_{n} \rightarrow 0$ implies $R_{n+1}-$ $R_{n} \rightarrow 0$.

\footnotetext{
${ }^{6}$ Knopp, p. 124.

7 Knopp, p. 285.
}

Theorem 5. If $S$ converges, $R=-1$ and $\left(1+R_{n+1}\right) /$ $\left(1+R_{n}\right)>1$, then $T$ converges with greater rapidity than $S$.

Proof: Since $R=-1$, we have $R_{n}<0$ for $n$ sufficiently large. Hence $R_{n} R_{n-1}>0$ and $1-R_{n}>1$. From theorem 2 it follows that $T$ converges so that $b_{n} \rightarrow 0$. Since $\left(1+R_{n+1}\right) /\left(1+R_{n}\right) \rightarrow 1$, it is positive for $n$ sufficiently large and

$$
\left(1+R_{n+1}\right)\left(1+R_{n-1}\right)=\frac{1+R_{n+1}}{1+R_{n}} \cdot \frac{1+R_{n-1}}{1+R_{n}}\left(1+R_{n}\right)^{2}>0,
$$

also

$$
\frac{\left(R_{n+1}-R_{n}\right)}{1+R_{n}}=\frac{1+R_{n+1}}{1+R_{n}}-1 \rightarrow 0 .
$$

Now

$$
\begin{aligned}
\left|\frac{b_{n}+b_{n+1}}{a_{n}+a_{n+1}}\right| & <\left|\frac{b_{n}}{a_{n}+a_{n+1}}\right|+\left|\frac{b_{n+1}}{a_{n}+a_{n+1}}\right| \\
& =\left|\frac{P_{n}}{1+R_{n+1}}\right|+\left|\frac{R_{n+1} P_{n+1}}{1+R_{n+1}}\right| \rightarrow 0,
\end{aligned}
$$

since

$\left|\frac{P_{n}}{1+R_{n+1}}\right|=\left|\frac{R_{n+1}-R_{n}}{1+R_{n}}\right|\left|\frac{1+R_{n}}{1+R_{n+1}}\right| \frac{1}{\left|1-R_{n+1}\right|\left|1-R_{n}\right| .} \rightarrow 0$,

and

$$
\left|\frac{R_{n+1} P_{n+1}}{1+R_{n+1}}\right|=\frac{\left|R_{n+1}\right|}{\left|1-R_{n+1}\right|\left|1-R_{n+2}\right|}\left|\frac{R_{n+2}-R_{n+1}}{1+R_{n+1}}\right| \rightarrow 0 .
$$

We may now apply Lemma 2.

Remarks. The condition $\left(1+R_{n+1}\right) /\left(1+R_{n}\right) \rightarrow 1$ requires that, for $n$ sufficiently large, $\left(1+R_{n}\right)$ be of constant sign. Since $S$ converges, this means that $R_{n}>-1$ for all sufficiently large $n$ so that terms decrease monotonically in magnitude. The converse is, however, not true. The condition can, of course, also be stated as $\left(R_{n+1}-R_{n}\right) /\left(1+R_{n}\right) \rightarrow 0$.

Theorem 6. If $S$ converges with $R_{n}>0$ and $Q_{n}>$ $K>0$ for $n$ sufficiently large, then $T$ converges. If, in addition, $n^{2}\left(R_{n+1}-R_{n}\right)$ it bounded as $n \rightarrow \infty$ then $T$ converges no less rapidly than $S$. If, further, $n^{2}\left(R_{n+1}-R_{n}\right) \rightarrow 0$ as $n \rightarrow \infty$ then $T$ converges more rapidly than $S$.

Proof: $\mathrm{By}$ hypothesis, for $n$ sufficiently large, $Q_{n}=n\left(1-R_{n}\right)>K$. Hence $1-R_{n}>K / n$ and $R_{n}<1-$ $K / n<1$. Since $R_{n}>0$, this means that terms of $S$ that correspond to sufficiently large $n$ are of like sign and monotonically decrease in magnitude. Since $S$ converges, we therefore have $n a_{n} \rightarrow 0$ (see footnote 6$)$. Hence $\left|a_{n} /\left(1-R_{n}\right)\right|=\left|n a_{n} / Q_{n}\right|<\left|n a_{n}\right| \mid K \rightarrow 0$. Therefore, by (7), $T_{n} \sim S_{n} \rightarrow S$.

Furthermore,

$$
\begin{aligned}
\left|P_{n}\right| & =\frac{\left|R_{n+1}-R_{n}\right|}{\left|1-R_{n+1}\right|\left|1-R_{n}\right|}=\frac{n(n+1)\left|R_{n+1}-R_{n}\right|}{\left|Q_{n} Q_{n+1}\right|} \\
& <\frac{n(n+1)\left|R_{n+1}-R_{n}\right|}{K^{2}},
\end{aligned}
$$


and is therefore bounded $($ or $\rightarrow 0)$ if $n^{2}\left(R_{n+1}-R_{n}\right)$ is bounded $($ or $\rightarrow 0$ ).

We may now apply Lemma 1.

Remarks. The boundedness of $n^{2}\left(R_{n+1}-R_{n}\right)$ requires that $R$ exist since it assures convergence of the series $\sum_{1}^{\infty}\left(R_{n+1}-R_{n}\right)$, whose partial sums are $R_{n+1}-R_{1}$. It does not, however, exclude the value $R=1$. The condition may also be stated as $\left[n Q_{n+1}-(n+1) Q_{n}\right]$ is bounded (or $\rightarrow 0$ ).

Theorem \%. If $S$ converges and $C$ exists, then $T$ converges. If, in addition, $n^{2}\left(R_{n+1}-R_{n}\right)$ is bounded as $n \rightarrow \infty$ then $T$ converges no less rapidly than $S$. If, further, $n^{2}\left(R_{n+1}-R_{n}\right) \rightarrow 0$ as $n \rightarrow \infty$ then $T$ converges more rapidly than $S$.

Proof: Since $Q_{n}=n\left(1-R_{n}\right) \rightarrow Q$, we have $1-R_{n} \rightarrow 0$. Hence $R_{n}>0$ for $n$ sufficiently large. Since $S$ converges, $Q \geq 1$ and thus $Q_{n}>\frac{1}{2}>0$ for $n$ sufficiently large. We now apply Theorem 6 .

Theorem 8. If $S$ converges, Q exists $\neq 1$, and $n\left(Q_{n}-\right.$ $\left.Q_{n-1}\right) \rightarrow 0$ as $n \rightarrow \infty$, then the series $U=\sum_{0}^{\infty} u_{n}$ with $\left.u_{n}=\left(Q b_{n}-a_{n}\right) / Q-1\right)$ converges more rapidly than $S$ and has the same sum.

Proof: From theorems (1) and (7), we have, with the present hypothesis that $T$ converges and $T=S$. Hence

$$
U_{n}=\sum_{0}^{n} u_{m}=\frac{Q T_{n}-S_{n}}{Q-1} \rightarrow \frac{Q S-S}{Q-1}=S .
$$

also

$$
\begin{aligned}
P_{n}=\frac{b_{n}}{a_{n}} & =\frac{1}{1-R_{n+1}}-\frac{1}{1-R_{n}}=\frac{n+1}{Q_{n+1}}-\frac{n}{Q_{n}} \\
& =\frac{1}{Q_{n}}-\frac{(n+1)\left(Q_{n+1}-Q_{n}\right)}{Q_{n} Q_{n+1}} \rightarrow \frac{1}{Q},
\end{aligned}
$$

since $Q \geq 1$ if $S$ converges. Hence

$$
\frac{u_{n}}{a_{n}}=\frac{Q P_{n}-1}{Q-1} \rightarrow 0 .
$$

Moreover, as shown in the proof of the previous theorem, existence of $Q$ implies $P_{n}>0$ for $n$ sufficiently large. We may thus use Lemma 1 to complete the proof.

Remarks. If any transformation or set of transformations leads from a convergent series $S$ to a convergent series $V=\sum_{0}^{\infty} v_{n}$, which has the same sum and if $v_{n} / a_{n} \rightarrow L \neq 1$ as $n \rightarrow \infty$, then the series $\mathrm{Z}=\sum_{0}^{\infty} z_{n}$ with $z_{n}=\left(v_{n}-L a_{n}\right) /(1-L)$ converges and has the sum $S$. Z Z may converge more rapidly than $S$. This will certainly be the case, since $z_{n} / a_{n} \rightarrow 0$, if $\left|1+R_{n}\right|>$ $K>0$ and $R_{n} R_{n-1}\left(1+R_{n+1}\right)\left(1+R_{n-1}\right)>0$ in view of Lemma 3 . The previous theorem is a special case of this. As another, and simpler case, consider a series $S$ such that, for a fixed integer $k, a_{n+k} / a_{n} \rightarrow L \neq 1$. Then we may take the $S$ series displaced $k$ terms for
V. That is, we take

$$
Z=\sum_{0}^{k-1} \frac{a_{n}}{1-L}+\sum_{k}^{\infty} \frac{a_{n}-L a_{n-k}}{1-L} .
$$

We shall call this simple transformation the "Ratio" transformation and use it as one of the comparison transformations in a later section. In particular, if $R$ exists $\neq 1$, we may use the Ratio transformation with $k=1$ and $L=\dot{R}$. Obviously, we may always reduce the transformation to this particular case by breaking the original series into $k$ subseries. Other applications of the general idea will be encountered in examples 4 and 5 , where it proves helpful, and in example 2, where it does not appear to be of assistance.

In attempting numerical use of the Ratio transformation or of the $U$ transformation of theorem 8, we are handicapped in many cases by not knowing the constants $R, Q, k$, or $L$, which appear in the formulae, even if we are certain that necessary conditions are met. We may write the Ratio transformation for $k=1$ as

$$
Z_{n-1}=\frac{S_{n}-R S_{n-1}}{1-R}
$$

In this form, if we approximate $R$ by $R_{n}$, we obtain the $T$ transformation:

$$
\begin{aligned}
T_{n-1} & =\frac{S_{n}-R_{n} S_{n-1}}{1-R_{n}} \\
& =S_{n-1}+\frac{S_{n}-S_{n-1}}{1-R_{n}}=S_{n-1}+\frac{a_{n}}{1-R_{n}} .
\end{aligned}
$$

Similarly, noting that $P_{n} \rightarrow 1 / Q$ in certain cases, we may approximate $1 / Q$ in the expression for $U_{n}$ by $P_{*}$. to obtain the $W$ transformation given by (17).

Both the $T$ transformation and the $W$ transformation have the advantage of being calculable without knowledge of an analytic expression for the general term. Each term of the former is completely determined by the values of a corresponding group of three successive terms of $S$, while four such terms are needed to calculate each term of $W$. Where the initial portion of the $S$ series has suitable properties, use of the $T$ series may produce the sum with sufficient accuracy, even though it may not be suitable when terms far out must be considered. Otherwise, at the expense of increased complexity, we may use the $W$ series. There are, of course, conditions under which neither transformation is of value. There are also peculiar cases where the $T$ transformation is usable but not the $W$. Examples of such conditions are included in the latter part of this section.

Theorem 9. If $S$ converges, $Q$ exists $\neq 1$, $n\left(Q_{n}-Q_{n-1}\right) \rightarrow 0$, and $n\left[(n+1) \quad\left(Q_{n+1}-Q_{n}\right)-n\left(Q_{n}-\right.\right.$ $\left.\left.Q_{n-1}\right)\right] \rightarrow 0$, then $W$ converges more rapidly than $S$ and has the same sum.

Proof: With the given hypothesis, the proof of theorem 8 shows that $P_{n} \rightarrow 1 / Q \neq 1$ and that $T_{n} \rightarrow S$. Hence, from (16), $W_{n} \rightarrow S$. Also, $Q_{n} \neq 0$ and $Q_{n}-$ $1 \neq 0$ for $n$ sufficiently large since $Q \neq 1$ by hypothesis and $Q \neq 0$ since $S$ converges. ${ }^{7}$ Hence

\footnotetext{
${ }^{7}$ Knopp, p. 285.
} 


$$
\begin{aligned}
\frac{W_{n}}{a_{n}} & =\frac{1-R_{n+1}}{1-2 R_{n+1}+R_{n} R_{n+1}}-\frac{1-R_{n-1}}{1-2 R_{n}+R_{n} R_{n-1}} \\
& =\frac{n\left(Q_{n}-Q_{n-1}\right)\left[2(n+1)\left(Q_{n+1}-Q_{n}\right)+\left(Q_{n}-2 Q_{n+1}\right)\right]-n Q_{n}\left[(n+1)\left(Q_{n+1}-Q_{n}\right)-n\left(Q_{n}-Q_{n-1}\right)\right]}{\left[(n+1)\left(Q_{n+1}-Q_{n}\right)+Q_{n+1}\left(Q_{n}-1\right)\right]\left[n\left(Q_{n}-Q_{n-1}\right)+Q_{n}\left(Q_{n-1}-1\right)\right]} .
\end{aligned}
$$

with the given hypothesis. Also, as shown in the proof of Theorem 7, existence of $Q$ implies $P_{n}>0$ for sufficiently large $n$ so that we may apply Lemma 1 .

Theorem 10. If the hypothesis of theorem 3, theorem 4 , or theorem 5 holds then $W$ converges more rapidly than $S$ and has the same sum.

Proof: The proofs of the theorems referred to show that, for any of the conditions stated, $P_{n} \rightarrow 0$ and $T$ converges more rapidly than $S$. Theorem 1 shows that $T=S$. We thus have $1-P_{n} \rightarrow 1$ and

$$
W_{n}=\frac{T_{n}-P_{n} S_{n}}{1-P_{n}} \sim T_{n} \rightarrow S,
$$

while

$$
\frac{S-W_{n}}{S-S_{n}}=\frac{\left(S-T_{n}\right) /\left(S-S_{n}\right)-P_{n}}{1-P_{n}} \rightarrow 0 .
$$

Theorem 11. If $S$ is an infinite convergent series and

$$
R_{n}=\alpha_{0}+\frac{\alpha_{1}}{n}+\frac{\alpha_{2}}{n^{2}}+\frac{\alpha_{3}}{n^{3}}+\ldots,
$$

is valid for all $n \geq N$ where the $\alpha_{i}$ and $N$ are constants, then $T$ converges more rapidly than $S$ if $\alpha_{0} \neq 1$ and with the same rapidity as $S$ if $\alpha_{0}=1$. If $\alpha_{i}=0$ for all $i>0$, then $T$ terminates with less than $N$ terms. Otherwise, $T$ is an infinite series with ratio of each term to its predecessor given by

$$
F_{n}=\frac{b_{n}}{b_{n-1}}=\beta_{0}+\frac{\beta_{1}}{n}+\frac{\beta_{2}}{n^{2}}+\frac{\beta_{3}}{n_{3}}+\ldots
$$

valid for all $n$ sufficiently large, where the $\beta_{i}$ are constants and not all $\beta_{i}=0$ for $i>0$. Moreover, $\beta_{0}=\alpha_{0}$. In addition, $\beta_{1}=\alpha_{1}$ if $\alpha_{0}=0$ or 1 and $\beta_{2}=\alpha_{2}$ if $\alpha_{0}+1$.

Proof: Let us write

$$
R(x)=\alpha_{0}+\alpha_{1} x+\alpha_{2} x^{2}+\alpha_{3} x^{4}+\ldots .
$$

This converges for $x=1 / N$ by hypothesis. It, therefore, converges for $|x|<1 / N$ and defines an analytic function in this range. Also, for $n \geq N$, $R_{n}=R(1 / n)$. We have

$$
\begin{aligned}
R_{-}(x)= & R[x /(1-x)]=\alpha_{0}+\alpha_{1} x+\left(\alpha_{2}+\alpha_{1}\right) x^{2}+ \\
& \left(\alpha_{3}+2 \alpha_{2}+\alpha_{1}\right) x^{3} \ldots \\
R_{+}(x)= & R[x /(1+x)]=\alpha_{0}+\alpha_{1} x+\left(\alpha_{2}-\alpha_{1}\right) x^{2}+ \\
& \left(\alpha_{3}-2 \alpha_{2}+\alpha_{1}\right) x^{3} \ldots
\end{aligned}
$$

valid for $|x|<1 /(N+1),{ }^{8}$ and $R_{n-1}=R_{-}(1 / n)$ and $R_{n+1}=R_{+}(1 / n)$ at least for $n>N+1$.

If $\alpha_{0} \neq 0,-1$, or 1 then, since $R(1 / n) \rightarrow \alpha_{0}$ as $n \rightarrow \infty$, the hypothesis of theorem 3 is satisfied and $T$ converges more rapidly than $S$.

If $\alpha_{0}=0$, not all $\alpha_{i}=0$ since this would make $R_{n}=0$ for all $n>N$ and $\mathrm{S}$ would terminate contrary to hypothesis. If $k$ is the smallest value of $i$ for which $\alpha_{i} \neq 0$, then $n^{k} R_{n}=\mathrm{R}(x) / x^{k}$ for $x=1 / n$ and approaches $\alpha_{k}$ whence all $R_{n}$ have the same sign, namely that of $\alpha_{k}$, for $n$ sufficiently large. Therefore, from theorem 4 , it follows that $T$ converges more rapidly than $S$.

If $\alpha_{0}=-1$, not all $\alpha_{i}=0$ since this would make $R_{n}=-1$ for all $n \geq N$, which is impossible since $\mathrm{S}$ converges. Again, let $k$ be the smallest value of $i$ for which $\alpha_{i} \neq 0$. Then $[1+R(x)] / x^{k} \rightarrow \alpha_{k}$ and

$$
\frac{1+R_{n+1}}{1+R_{n}}=\frac{(n+1)^{k}\left(1+R_{n+1}\right)}{n^{k}\left(1+R_{n}\right)} \cdot\left(\frac{n}{n+1}\right)^{k} \sim \frac{\alpha_{k}}{\alpha_{k}}\left(1+\frac{1}{n}\right)^{-k} \rightarrow 1
$$

and theorem 5 shows that $T$ converges more rapidly than $S$.

If $\alpha_{0}=1$, we have

$$
Q(x)=[1-R(x)] / x=-\alpha_{1}-\alpha_{2} x-\alpha_{3} x^{2}-\alpha_{4} x^{3}-\ldots
$$

and

$$
\begin{aligned}
Q_{-}(x) & =Q[x /(1-x)] \\
& =-\alpha_{1}-\alpha_{2} x-\left(\alpha_{3}+\alpha_{2}\right) x^{2}-\left(\alpha_{4}+2 \alpha_{3}+\alpha_{2}\right) x^{3}-\ldots
\end{aligned}
$$

valid for $x<1 /(N+1)$ and $Q_{n}=n\left(1-R_{n}\right)=Q(1 / n)$ while $Q_{n-1}=Q_{-}(1 / n)$ at least for $n>N+1$. Hence

$$
\begin{aligned}
q(x) & =\left[Q(x)-Q_{-}(x)\right] / x \\
& =\alpha_{2} x+\left(2 \alpha_{3}+\alpha_{2}\right) x^{2}+\left(3 \alpha_{4}+3 \alpha_{3}+\alpha_{2}\right) x^{3}+\ldots
\end{aligned}
$$

and $n\left(Q_{n}-Q_{n-1}\right)=q(1 / n) \rightarrow 0$ while $Q_{n} \rightarrow-\alpha_{1}$. Since $S$ converges, $\alpha_{1}<-1^{9}$ or $Q=-\alpha_{1}>1$. The proof of theorem 8 indicates that, under these conditions, $P_{n} \rightarrow 1 / Q>0$. For $n$ sufficiently large, we thus have $3 / 2 Q>P_{n}>1 / 2 Q$ and, by Lemma 1 , the series $T$ converges with the same order of rapidity as $S$.

If all $\alpha_{i}=0$ for $i>0$, then $R_{\mathrm{n}}=\alpha_{0}$ for all $n \geqslant N$. We cannot have $\alpha_{0}=1$ in this case, since $S$ converges. Hence $1-\alpha_{0} \neq 0$ and, from $(9), b_{n}=a_{n}\left[1 /\left(1-\alpha_{0}\right)\right.$ $\left.1 /\left(1-\alpha_{0}\right)\right]=0$ for all $n \geqslant N$ and $T$ terminates with less than $N$ terms.

Conversely, if $T$ terminates with $K$ terms, then $b_{n}=0$ for all $n>K$. But if $a_{n}=0$ for any $n \geqslant N$, the finiteness of the $P_{n}$ makes all subsequent $a_{n}=0$ so that $S$ terminates contrary to hypothesis. Hence $a_{n} \neq 0$ for $n \geqslant N$. We therefore require $P_{n}=0$ for all $n \geqslant M$, where $M$ is the larger of $K+1$ and $N$. From (13) it follows that this implies $R_{n+1}-R_{n}=0$ for all $n \geqslant M$ so that $R_{n}$ is constant in this range $\circ$ Knopp, p. 288. 
and $R(x)$ is equal to $R_{0}(x)=R_{M}+0 x+0 x^{2}+0 x^{3}+$. . for the null sequence $x=1 / n$. Hence all $\alpha_{i}=0$ for $i>0 .{ }^{10}$ If, therefore, not all $\alpha_{i}=0$ for $i>0, T$ is an infinite series. If not all $\alpha_{i}=0$ for $i>0$, let $k$ be the least value of $i>0$ for which $\alpha_{i} \neq 0$. Then

$$
\begin{aligned}
R_{+}(x)-R(x)= & -k \alpha_{k} x^{k+1}-(k+1)\left(\alpha_{k+1}-k \alpha_{k} / 2\right) x^{k+2}- \\
& (k+2)\left[\alpha_{k+2}-(k+1) \alpha_{k+1} / 2+\right. \\
& \left.k(k+1) \alpha_{k} / 3 !\right] x^{k+3}+\ldots
\end{aligned}
$$

and

$$
\begin{aligned}
{[1-R(x)]\left[1-R_{+}(x)\right] } & =\left(1-\alpha_{0}\right)^{2}-2\left(1-\alpha_{0}\right) \alpha_{1} x-\left(1-\alpha_{0}\right) \\
& \times\left(2 \alpha_{2}-\alpha_{1}-\frac{\alpha_{1}^{2}}{1-\alpha_{0}}\right) x^{2}-\ldots
\end{aligned}
$$

all valid for $|x|<1 /(N+1)$. Furthermore, we have

$$
\begin{aligned}
P(x)= & \frac{R_{+}(x)-R(x)}{[1-R(x)]\left[1-R_{+}(x)\right]} \\
= & -\frac{k \alpha_{k} x^{k+1}}{\left(1-\alpha_{0}\right)^{2}}-\frac{(k+1)\left(\alpha_{k+1}-k \alpha_{k} / 2\right)}{\left(1-\alpha_{0}\right)^{2}} x^{k+2}- \\
& \frac{(k+2)\left[\alpha_{k+2}-(k+1) \alpha_{k+1} / 2+k(k+1) \alpha_{k} / 6\right]}{\left(1-\alpha_{0}\right)^{2}} x^{k+3} \ldots
\end{aligned}
$$

for $k>2$; and

$$
\begin{aligned}
= & -\frac{2 \alpha_{2} x^{3}}{\left(1-\alpha_{0}\right)^{2}}-\frac{3\left(\alpha_{3}-\alpha_{2}\right) x^{4}}{\left(1-\alpha_{0}\right)^{2}}- \\
& \frac{2 x^{5}}{\left(1-\alpha_{0}\right)^{2}}\left[2 \alpha_{4}-3 \alpha_{3}+2 \alpha_{2}+\frac{2 \alpha_{2}^{2}}{1-\alpha_{0}}\right]-\cdots
\end{aligned}
$$

for $k=2$, noting that we cannot have $\alpha_{0}=1$ in these cases. For $k=1, \alpha_{0} \neq 1$, this becomes

$$
\begin{aligned}
P(x)= & -\frac{\alpha_{1} x^{2}}{\left(1-\alpha_{0}\right)^{2}}-\frac{1}{\left(1-\alpha_{0}\right)^{2}}\left[2 \alpha_{2}-\alpha_{1}+\frac{2 \alpha_{1}^{2}}{1-\alpha_{0}}\right] x^{3}- \\
& \frac{1}{\left(1-\alpha_{0}\right)^{2}}\left[3 \alpha_{3}-3 \alpha_{2}+\alpha_{1}+\frac{3 \alpha_{1}\left(2 \alpha_{2}-\alpha_{1}\right)}{1-\alpha_{0}}+\right. \\
& \left.\frac{3 \alpha_{1}^{3}}{\left(1-\alpha_{0}\right)^{2}}\right] x^{4}-\ldots
\end{aligned}
$$

while, for $\alpha_{0}=1$ (and, therefore, $k=1$ ),

$$
\begin{aligned}
P(x)= & -\frac{1}{\alpha_{1}}-\frac{1}{\alpha_{1}}\left[\frac{\alpha_{3}}{\alpha_{1}}-\frac{\alpha_{2}^{2}}{\alpha_{1}^{2}}\right] x^{2}- \\
& \frac{1}{\alpha_{1}}\left[\frac{2 \alpha_{4}}{\alpha_{1}}-\frac{\alpha_{3}}{\alpha_{1}}-\frac{4 \alpha_{2} \alpha_{3}}{\alpha_{1}^{2}}+\frac{2 \alpha_{2}^{3}}{\alpha_{1}^{3}}+\frac{\alpha_{2}^{2}}{\alpha_{1}^{2}}\right] x^{3}-\ldots
\end{aligned}
$$

all valid for sufficiently small $|x|{ }^{11} \quad$ Then

\footnotetext{
${ }^{30}$ Knopp, p. 172.
}

${ }_{11}$ Knopp, p. 182.

$$
\begin{aligned}
P_{-}(x) & =P\left[\frac{x}{1-x}\right] \\
& =-\frac{k \alpha_{k} x^{k+1}}{\left(1-\alpha_{0}\right)^{2}}-\frac{(k+1)\left(\alpha_{k+1}+k \alpha_{k} / 2\right) x^{k+2}}{\left(1-\alpha_{0}\right)^{2}}-
\end{aligned}
$$$$
\frac{(k+2)\left[\alpha_{k+2}+(k+1) \alpha_{k+1} / 2+k(k+1) \alpha_{k} / 6\right] x^{k+3}}{\left(1-\alpha_{0}\right)^{2}}-\ldots
$$

for $k>2$; and

$$
\begin{aligned}
= & -\frac{2 \alpha_{2} x^{3}}{\left(1-\alpha_{0}\right)^{2}}-\frac{3\left(\alpha_{3}+\alpha_{2}\right) x^{4}}{\left(1-\alpha_{0}\right)^{2}}- \\
& \frac{2 x^{5}}{\left(1-\alpha_{0}\right)^{2}}\left[2 \alpha_{4}+3 \alpha_{3}+2 \alpha_{2}+\frac{2 \alpha_{2}^{2}}{1-\alpha_{0}}\right]-\cdots
\end{aligned}
$$

for $k=2$; and

$$
\begin{aligned}
P_{-}(x)= & -\frac{\alpha_{1} x^{2}}{\left(1-\alpha_{0}\right)^{2}}-\frac{1}{\left(1-\alpha_{0}\right)^{2}}\left[2 \alpha_{2}+\alpha_{1}+\frac{2 \alpha_{1}^{2}}{1-\alpha_{0}}\right] x^{3}- \\
& \frac{1}{\left(1-\alpha_{0}\right)^{2}}\left[3 \alpha_{3}+3 \alpha_{2}+\alpha_{1}+\frac{3 \alpha_{1}\left(2 \alpha_{2}+\alpha_{1}\right)}{1-\alpha_{0}}+\right. \\
& \left.\frac{3 \alpha_{1}^{3}}{\left(1-\alpha_{0}\right)^{2}}\right] x^{4}-\cdots
\end{aligned}
$$

for $k=1, \quad \alpha_{0} \neq 1$. Finally, for $\alpha_{0}=1$,

$$
\begin{aligned}
P_{-}(x) & =-\frac{1}{\alpha_{1}}-\frac{1}{\alpha_{1}}\left[\frac{\alpha_{3}}{\alpha_{1}}-\frac{\alpha_{2}^{2}}{\alpha_{1}^{2}}\right] x^{2}- \\
& \frac{1}{\alpha_{1}}\left[\frac{2 \alpha_{4}}{\alpha_{1}}+\frac{\alpha_{3}}{\alpha_{1}}-\frac{4 \alpha_{2} \alpha_{3}}{\alpha_{1}^{2}}+\frac{2 \alpha_{2}^{3}}{\alpha_{1}^{3}}-\frac{\alpha_{2}^{2}}{\alpha_{1}^{2}}\right] x^{3}-\cdots
\end{aligned}
$$

We thus get, for $|x|$ sufficiently small

$G(x)=\frac{P(x)}{P_{-}(x)}=1-(k+1) x+(k+1)\left[\frac{k+1}{2}-\frac{\alpha_{k+1}}{k \alpha_{k}}\right] x^{2}-\cdots$

for $k \neq 1$; and

$$
G(x)=1-2 x+2\left[1-\frac{\alpha_{2}}{\alpha_{1}}-\frac{\alpha_{1}}{1-\alpha_{0}}\right] x^{2}-\cdots
$$

for $k=1, \alpha_{0} \neq 1$; and

$$
G(x)=1+2\left[\frac{\alpha_{2}^{2}}{\alpha_{1}^{2}}-\frac{\alpha_{3}}{\alpha_{1}}\right] x^{3}-\cdots
$$

for $\alpha_{0}=1$. We may now write

$$
F(x)=G(x) R(x)=\beta_{0}+\beta_{1} x+\beta_{2} x^{2}+\beta_{3} x^{3}+\ldots .
$$

where $\beta_{0}=\alpha_{0}$ in all cases; $\beta_{1}=\alpha_{1}$ when $\alpha_{0}=0$ or 1 ; and $\beta_{2}=\alpha_{2}$ when $\alpha_{0}=1$. For $\alpha_{0} \neq 0, k \neq 1$, we have $\beta_{1}=$ $-(k+1) \alpha_{0} \neq 0$. For $\alpha_{0} \neq 0$ or $1, k=1$, we have $\beta_{1}=\alpha_{1}-2 \alpha_{0} \neq 0$ unless $\alpha_{1}=2 \alpha_{0}$. In the latter case, we find $\beta_{2}=-2 \alpha_{0}\left(1+\alpha_{0}\right) /\left(1-\alpha_{0}\right) \neq 0$ unless $\alpha_{0}=-1$. However, we cannot have $\left|R_{n}\right|>1$ for all sufficiently large $n$, and hence $1+R_{n}$ cannot become and stay negative. But $n\left(1+R_{n}\right) \rightarrow \alpha_{1}$ if $\alpha_{0}=-1$. Hence we must have $\alpha_{1}>0$ and thus $\alpha_{1} \neq-2=2 \alpha_{0}$ so that $\beta_{1} \neq 0$. For $\alpha_{0}=0$, we have $\beta_{k}=\alpha_{k} \neq 0$. In all cases, 
therefore, not all the $\beta_{i}=0$ for $i>0$. But, by inspection, $F_{n}=F(1 / n)$ for $n$ sufficiently large. Hence our proof is complete.

Theorem 12. If $S$ is an infinite convergent series and

$$
R_{n}=\alpha_{0}+\frac{\alpha_{1}}{n}+\frac{\alpha_{2}}{n^{2}}+\frac{\alpha_{3}}{n^{3}}+\ldots
$$

is valid for all $n \geqslant N$ where the $\alpha_{i}$ and $N$ are constants, then $W$ converges more rapidly than $S$ and has the same sum. If $\alpha_{i}=0$ for all $i>0$ or if $\alpha_{0}=1$ and $\alpha_{i}=\left(\alpha_{2} / \alpha_{1}\right)^{i-2} \alpha_{2}$ for all $i>2$, then $W$ terminates with less than $N$ terms. Otherwise $W$ is an infinite series with ratio of each term to its predecessor given by

$$
f_{n}=\frac{w_{n}}{w_{n-1}}=\gamma_{0}+\frac{\gamma_{1}}{n}+\frac{\gamma_{2}}{n^{2}}+\frac{\gamma_{3}}{u^{3}}+\ldots
$$

valid for all sufficiently large $n$, where the $\gamma_{i}$ are constants and not all $\gamma_{i}=0$ for $i>0$. Moreover, $\gamma_{0}=\alpha_{0}$ and, if $\alpha_{0}=0, \gamma_{1}=\alpha_{1}$.

Proof: We shall use a large part of the proof of theorem 11. We note that

$q_{+}(x)=q[x /(1+x)]=\alpha_{2} x+2 \alpha_{3} x^{2}+\left(3 \alpha_{4}-\alpha_{3}\right) x^{3}+\ldots$

so that

$$
H(x)=\left[q_{+}(x)-q(x)\right] / x=-\alpha_{2} x-\left(4 \alpha_{3}+\alpha_{3}\right) x^{2}-\ldots
$$

valid for $|x|<1 /(N+1)$. Therefore,

$$
n\left[(n+1)\left(Q_{n+1}-Q_{n}\right)-n\left(Q_{n}-Q_{n-1}\right)\right]=H(1 / n) \rightarrow 0 .
$$

This, coupled with other data from the proof of the theorem 11, shows that the hypothesis of either theorem 9 or theorem 10 is satisfied so that $W$ converges more rapidly than $S$ and has the same sum.

If all $\alpha_{i}=0$ for $i>0$, we have $R_{n}=\alpha_{0}$ for all $n \geqslant N$ and $\alpha_{0} \neq 1$ since $S$ converges. Hence $1-\alpha_{0} \neq 0$ and, using $(19), w_{n} / a_{n}=1 /\left(1-\alpha_{0}\right)-1 /\left(1-\alpha_{0}\right)=0$ for all $n \geqslant N$, so that $W$ terminates with less than $N$ terms.

If all $\alpha_{i}=\left(\alpha_{2} / \alpha_{1}\right)^{i-2} \alpha_{2}$ for $i>2$ and $\alpha_{0}=1$, we have $R_{n}=1+\alpha_{1} /\left(n-\alpha_{2} / \alpha_{1}\right)$ for all $n \geqslant N$. Using (19), $w_{n} / a_{n}=-\left(n-\alpha_{2} / \alpha_{1}\right) /\left(1+\alpha_{1}\right)+\left(n-\alpha_{2} / \alpha_{1}\right) /\left(1+\alpha_{1}\right)=0$ for all $n>N$ since $-\alpha_{1}>1$ and $1+\alpha_{1} \neq 0$ if $S$ converges. Thus $W$ terminates with less than $N$ terms.

Let us now consider $\alpha_{0} \neq 1$ and not all $\alpha_{i}=0$ for $i>0$. If $k$ is the smallest index $i>0$ for which $\alpha_{i} \neq 0$, we obtain

$$
\begin{aligned}
A(x)= & {\left[1-R_{-}(x)\right]\left[R_{+}(x)-R(x)\right]-} \\
& {\left[1-R_{+}(x)\right]\left[R(x)-R_{-}(x)\right] } \\
= & \left(1-\alpha_{0}\right) k(k+1) \alpha_{k} x^{k+2}+ \\
& \left(1-\alpha_{0}\right)(k+1)(k+2) \alpha_{k+1} x^{k+3} \\
+ & \left(1-\alpha_{0}\right)(k+2)(k+3)\left[\alpha_{k+2}+k(k+1) \alpha_{k} / 12\right] x^{k+4}+ \\
& \cdots
\end{aligned}
$$

for $k \neq 2$; and

$$
\begin{aligned}
A(x)= & 6\left(1-\alpha_{0}\right) \alpha_{2} x^{4}+12\left(1-\alpha_{0}\right) \alpha_{3} x^{5}+ \\
& \left(1-\alpha_{0}\right)\left[20 \alpha_{4}+10 \alpha_{2}+\frac{2 \alpha_{2}^{2}}{1-\alpha_{0}}\right] x^{6}-\ldots
\end{aligned}
$$

for $k=2$; both valid for $|x|<1 /(N+1$.) But, from (19), $w_{n} / a_{n}=A_{n} / B_{n}$, where

$$
\begin{aligned}
A_{n}= & \left(1=R_{n+1}\right)\left(1-2 R_{n}+R_{n} R_{n-1}\right)- \\
& \left(1-R_{n-1}\right)\left(1-2 R_{n+1}+R_{n} R_{n+1}\right) \\
= & \left(1-R_{n-1}\right)\left(R_{n+1}-R_{n}\right)-\left(1-R_{n+1}\right)\left(R_{n}-R_{n-1}\right)
\end{aligned}
$$

and

$$
B_{n}=\left(1-2 R_{n}+R_{n} R_{n-1}\right)\left(1-2 R_{n+1}+R_{n} R_{n+1}\right) .
$$

Since $a_{n} \neq 0$ for $n \geqslant N$ as indicated in the proof of theorem 11, if $W$ terminates with $K$ terms, we must have $A_{n}=0$ for all $n \geqslant M$, where $M$ is the larger of $N$ and $K+1$. But $A(x)$ cannot vanish for all terms of the null sequence $x=1 / n, n \geqslant M$ since $\alpha_{k} \neq 0$. Hence $A_{n}=A(1 / n)$ cannot be zero for all $n \geqslant M$, and $W$ is an infinite series. Furthermore, we readily obtain

$$
\begin{aligned}
B(x)= & {\left[1-2 R(x)+R(x) R_{-}(x)\right]\left[1-2 R_{+}(x)+R(x) R_{+}(x)\right] } \\
= & \left(1-\alpha_{0}\right)^{4}-4\left(1-\alpha_{0}\right)^{3} \alpha_{1} x- \\
& {\left[4\left(1-\alpha_{0}\right)^{3} \alpha_{2}-2\left(1-\alpha_{0}\right)^{2} \alpha_{1}-6\left(1-\alpha_{0}\right)^{2} \alpha_{1}^{2}\right] x^{2}-\ldots }
\end{aligned}
$$

if $k=1$; and, for $k \neq 1$,

$$
\begin{aligned}
B(x)= & \left(1-\alpha_{0}\right)^{4}-4\left(1-\alpha_{0}\right)^{3} \alpha_{k} x^{k}- \\
& {\left[4\left(1-\alpha_{0}\right)^{3} \alpha_{k+1}-2\left(1-\alpha_{0}\right)^{2} k \alpha_{k}\right] x^{k+1}-\ldots }
\end{aligned}
$$

Thus, for $|x|$ sufficiently small,

$$
\begin{array}{r}
p(x)=\frac{A(x)}{B(x)}=\frac{x^{k+2}}{\left(1-\alpha_{0}\right)^{3}}\left[k(k+1) \alpha_{k}+(k+1)(k+2) \alpha_{k+1} x+\right. \\
\left.(k+2)(k+3)\left\{\alpha_{k+2}+\frac{k(k+1)}{12} \alpha_{k}\right\} x^{2}+\ldots\right]
\end{array}
$$

for $k>2$; and

$$
\begin{aligned}
p(x)= & \frac{x^{4}}{\left(1-\alpha_{0}\right)^{3}}\left[6 \alpha_{2}+12 \alpha_{3} x+\right. \\
& \left.\left(20 \alpha_{4}+10 \alpha_{2}+\frac{26 \alpha_{2}^{2}}{1-\alpha_{0}}\right) x^{2}+\ldots\right]
\end{aligned}
$$

for $k=2$; and

$$
\begin{aligned}
p(x)= & \frac{x^{3}}{\left(1-\alpha_{0}\right)^{3}}\left[2 \alpha_{1}+\left(6 \alpha_{2}-\frac{8 \alpha_{1}^{2}}{1-\alpha_{0}}\right) x+\left\{12 \alpha_{3}+2 \alpha_{1}+\right.\right. \\
& \left.\left.\left.\frac{32 \alpha_{1} \alpha_{2}}{1-\alpha_{0}}-\frac{4 \alpha_{1}^{2}}{\left(1-\alpha_{0}\right)^{2}}+\frac{20 \alpha_{1}^{3}}{\left(1-\alpha_{0}\right)^{2}}\right\} x^{2}\right\}+\ldots\right]
\end{aligned}
$$

for $k=1$. From these, we further get 


$$
\begin{aligned}
p_{-}(x)= & p\left[\frac{x}{1-x}\right]=\frac{x^{k+2}}{\left(1-\alpha_{0}\right)^{3}}\left[k(k+1) \alpha_{k}+\right. \\
& (k+1)(k+2)\left(\alpha_{k+1}+k \alpha_{k}\right) x+(k+2)(k+3) \\
& \left.\times\left\{\alpha_{k+2}+(k+1) \alpha_{k+1}+\frac{7 k(k+1) \alpha_{k}}{12}\right\} x^{2}+\ldots\right]
\end{aligned}
$$

or

$$
\begin{aligned}
p_{-}(x)= & \frac{x^{4}}{\left(1-\alpha_{0}\right)^{3}}\left[6 \alpha_{2}+12\left(\alpha_{3}+2 \alpha_{2}\right) x+\right. \\
& \left.20\left\{\alpha_{4}+2 \alpha_{3}+\frac{7 \alpha_{2}}{2}+\frac{13 \alpha_{2}^{2}}{10\left(1-\alpha_{0}\right)}\right\} x^{2}+\ldots\right]
\end{aligned}
$$

or

$$
\begin{aligned}
p_{-}(x)= & \frac{x^{3}}{\left(1-\alpha_{0}\right)^{3}}\left[2 \alpha_{1}+6\left\{\alpha_{2}+\alpha_{1}+\frac{4 \alpha_{1}^{2}}{3\left(1-\alpha_{0}\right)}\right\} x+\right. \\
& 12\left\{\alpha_{3}+2 \alpha_{2}+\frac{7 \alpha_{1}}{6}+\frac{8 \alpha_{1} \alpha_{2}}{3\left(1-\alpha_{0}\right)}+\frac{8 \alpha_{1}^{2}}{3\left(1-\alpha_{0}\right)}-\right. \\
& \left.\left.\frac{\alpha_{1}^{2}}{3\left(1-\alpha_{0}\right)^{2}}+\frac{5 \alpha_{1}^{3}}{3\left(1-\alpha_{0}\right)^{2}}\right\} x^{2}-\ldots\right]
\end{aligned}
$$

for $k>2, k=2$, and $k=1$, respectively. Hence, for sufficiently small $|x|$,

$g(x)=\frac{p(x)}{p_{-}(x)}=1-(k+2) x+(k+2)\left[\frac{k+1}{2}-\frac{\alpha_{k+1}}{k \alpha_{k}}\right] x^{2}-\ldots$

or

$$
g(x)=1-3 x+3\left[1-\frac{\alpha_{2}}{\alpha_{1}}-\frac{4 \alpha_{1}}{3\left(1-\alpha_{0}\right)}\right] x^{2}-\ldots
$$

for $k \neq 1$ and $k=1$, respectively. We may now write

$$
f(x)=g(x) R(x)=\gamma_{0}+\gamma_{2} x+\gamma_{2} x^{2}+\gamma_{2} x^{3}+\ldots .
$$

where $\gamma_{0}=\alpha_{0}$ in all cases and $\gamma_{1}=\alpha_{1}$ when $\alpha_{0}=0$. Also, for $\alpha_{0}=0, \gamma_{k}=\alpha_{k} \neq 0$. For $\alpha_{0} \neq 0, k \neq 1$, we have $\gamma_{1}=-(k+2) \alpha_{0} \neq 0$. For $\alpha_{0} \neq 0, k=1$, we have $\gamma_{1}=\alpha_{1}-3 \alpha_{0} \neq 0$ unless $\alpha_{1}=3 \alpha_{0}$. In the latter case, we find $\gamma_{2}=-6 \alpha_{0}\left(1+\alpha_{0}\right) /\left(1-\alpha_{0}\right) \neq 0$ unless $\alpha_{0}=-1$. However, if $\alpha_{0}=-1$, we must have $\alpha_{1}>0$, and hence $\alpha_{1} \neq-3=3 \alpha_{0}$ so that $\gamma_{1} \neq 0$. In all cases, therefore, not all the $\gamma_{i}=0$ for $i>0$. By inspection, $f_{n}=f(1 / n)$ for $n$ sufficiently large. Hence our proof for the case $\alpha_{0} \neq 1$ is complete.

Finally, let us consider $\alpha_{0}=1$ and not all $\alpha_{i}=$ $\left(\alpha_{2} / \alpha_{1}\right)^{i-2} \alpha_{2}$ for $i>2$. Let $k$ be the smallest index $i>2$ for which $\alpha_{i} \neq\left(\alpha_{2} / \alpha_{1}\right)^{i-2} \alpha_{2}$. Then we have

$$
R_{n}=1+\frac{\alpha_{1}}{m}+\frac{\delta_{k}}{m^{k}}+\frac{\delta_{k+1}}{m^{k+1}}+\ldots,
$$

where $m=n-\alpha_{2} / \alpha_{1}, \quad \delta_{i}=\alpha_{i}-\left(\alpha_{2} / \alpha_{1}\right)^{i-2} \alpha_{2}, \quad \delta_{k} \neq 0, \quad$ and $k>2$. If we now write

$$
r(y)=1+\alpha_{1} y+\delta_{k} y^{k}+\delta_{k+1} y^{k+1}+\ldots,
$$

then $r(y)$ converges for $y=1 / M$, where $M=N-\alpha_{2} / \alpha_{1}$ and hence for $|y|<1 / M$ and defines an analytic function of $y$ in this range. Also, for $m>M$, we have $R_{n}=r(1 / m)$ where $m=n-\alpha_{2} / \alpha_{1}$. We will, however, find it more convenient to use the function

$$
D(y)=\alpha_{1}+\delta_{k} y^{k-1}+\delta_{k+1} y^{k}+\ldots .
$$

By substitution, we also get,

$$
\begin{aligned}
D_{-}(y)= & D[y /(1-y)]=\alpha_{1}+\delta_{k} y^{k-1}+\left[\delta_{k+1}+(k-1) \delta_{k}\right] y^{k}+ \\
& {\left[\delta_{k+1}+k \delta_{k+1}+k(k-1) \delta_{k} / 2\right] y^{k+1}+\ldots }
\end{aligned}
$$

and

$$
\begin{aligned}
D_{+}(y)= & D[y /(1+y)]=\alpha_{1}+\delta_{k} y^{k-1}+\left[\delta_{k+1}-(k-1) \delta_{k}\right] y^{k}+ \\
& {\left[\delta_{k+2}-k \delta_{k+1}+k(k-1) \delta_{k} / 2\right] y^{k+1}+\ldots }
\end{aligned}
$$

valid for $|y|<1 /(M+1)$. It is obviously that

and

$$
\begin{aligned}
r(y) & =1+y D(y) \\
r_{-}(y) & =r[y /(1-y)]=1+y D_{-}(y) /(1-y)
\end{aligned}
$$

$$
r_{+}(y)=r[y /(1+y)]=1+y D_{+}(y) /(1+y) .
$$

Thus

$$
\begin{aligned}
a(y)= & {\left[1-r_{-}(y)\right]\left[r_{+}(y)-r(y)\right]-\left[1-r_{+}(y)\right]\left[r(y)-r_{-}(y)\right] } \\
= & \left\{D_{-}(y)\left[(1+y) D(y)-D_{+}(y)\right]-D_{+}(y)\left[D_{-}(y)-\right.\right. \\
& (1-y) D(y)]\} y^{2} /\left(1-y^{2}\right) \\
= & -\left[\alpha_{1}(k-1)(k-2) \delta_{k}+\alpha_{1} k(k-1) \delta_{k+1} y+\right. \\
& \ldots .] y^{k+3} /\left(1-y^{2}\right) .
\end{aligned}
$$

By inspection, $A_{n}=a(1 / n)$ and cannot vanish for all sufficiently large $n$ since $\alpha_{1}(k-1)(k-2) \delta_{k} \neq 0$. As previously stated, all $a_{n} \neq 0$ for $n>N$. Hence $w_{n}$ does not vanish for all sufficiently large $n$, and $W$ is an infinite series. Also,

$$
\begin{aligned}
b(y)= & {\left[1-2 r(y)+r(y) r_{-}(y)\right]\left[1-2 r_{+}(y)+r(y) r_{+}(y)\right] } \\
= & {\left[D_{-}(y)-(1-y) D(y)+y D(y) D_{-}(y)\right] \times } \\
& {\left[(1+y) D(y)-D_{+}(y)+y D(y) D_{+}(y)\right] y^{2} /\left(1-y^{2}\right) } \\
= & {\left[\alpha_{1}^{2}\left(1+\alpha_{1}\right)^{2}+2 \alpha_{1}\left(1-\alpha_{1}\right)\left(k+2 \alpha_{1}\right) \delta_{k} y^{k-1}+\right.} \\
& \ldots .] y^{4} /\left(1-y^{2}\right) .
\end{aligned}
$$

Thus

$$
\begin{aligned}
e(y)= & \frac{a(y)}{b(y)}=-\frac{(k-1)(k-2)}{\alpha_{1}\left(1+\alpha_{1}\right)^{2}} \delta_{k} y^{k-1}- \\
& \frac{k(k-1)}{\alpha_{1}\left(1+\alpha_{1}\right)^{2}} \delta_{k-1} y^{k}+\ldots,
\end{aligned}
$$

and

$$
\begin{aligned}
e_{-}(y) & =e\left[\frac{y}{1-y}\right]=-\frac{(k-1)(k-2)}{\alpha_{1}\left(1+\alpha_{1}\right)^{2}} \delta_{k} y^{k-1}- \\
& =\frac{(k-1)\left[k \delta_{k+1}+(k-1)(k-2) \delta_{k}\right]}{\alpha_{1}\left(1+\alpha_{1}\right)^{2}} y^{k}+\ldots,
\end{aligned}
$$


so that

$$
d(y)=e(y) / e_{-}(y)=1-(k-1) y+\ldots,
$$

and

$$
D(y)=d(y) r(y)=1+\left(\alpha_{1}+1-k\right) y+\ldots
$$

If

$$
f(x)=D\left[x /\left(1-\alpha_{2} x / \alpha_{1}\right)\right]=1+\left(\alpha_{1}+1-k\right) x+\ldots,
$$

then

$$
f_{n}=f(1 / n)=\gamma_{0}+\frac{\gamma_{1}}{n}+\frac{\gamma_{2}}{n^{2}}+\cdots
$$

where

$$
\gamma_{0}=\alpha_{0}(=1) \text { and } \gamma_{1}=\alpha_{1}+1-k<-k \text { since }-\alpha_{1}>1 \text {. }
$$

Remarks. The last theorem shows that, if $R_{n}$ is an analytic function of $1 / n$ for $n$ sufficiently large, then the $W$ transformation will yield a new series with the same sum but more rapidly convergent. Moreover, the transformation can be applied to the new series, if infinite, to get a third series with the same sum but still more rapidly convergent. The process can be repeated as often as desired to yield successive series, each of which converges more rapidly than its predecessor and each of which has the same sum as the original series. If $R \neq 1$, theorem 11 shows that the same statements hold for the simpler $T$ transformation. If $R=1$, an infinite set of transformed series may be obtained by the $T$ transformation, each of which has the same sum as the original series, but each will converge only with the same order of rapidity as the original.

Example 1:

$$
S=\log 2=1-\frac{1}{2}+\frac{1}{3}-\frac{1}{4}+\frac{1}{5}-\frac{1}{6}+\frac{1}{7}-\frac{1}{8}+\frac{1}{9} \cdots
$$

This series is very slowly convergent. To obtain 9 -place accuracy with this series would require use of $10^{9}$ terms were it used directly. Since, however, we have

$$
R_{n}=-\frac{n}{n+1}=-1+\frac{1}{n}-\frac{1}{n^{2}}+\cdots,
$$

for this series, it satisfies the hypothesis of theorem 11 and may be subjected to repeated $T$ transformations with increased rapidity of convergence at each step. Restricting ourselves to using only the first 9 terms of $S$, the successive transformed series are

$$
\begin{aligned}
& T=.6666666667+.333333333-.0095238095 \\
& \quad+.0039682540-.0020202020+.0011655012 \\
& \quad-.0007326007+.0004901961-\ldots .
\end{aligned}
$$

$$
\begin{aligned}
T^{\prime} & =.6925925926+.0006847183-.0001715546 \\
& +.0000575844-.0000234397+.0000109276 \\
T^{\prime \prime} & =.6931401274+.0000087420-.0000021874 \\
& +.0000006721-\ldots \\
T^{\prime \prime \prime} & =.6931471197+.0000000763-\ldots
\end{aligned}
$$

in which we have omitted the first step in each of the succeeding transformations since, obviously, the first term in each of the $T$ series is not smoothly related to the remainder of the terms. It would not have affected results were the transformation also applied to the first terms, the further series merely carrying additional terms of irregular form with the same terms as given following these. This initial irregularity is due to the difference in form for the first term in each transformation from later terms as shown by (9).

The error of stopping at a given place in any of the transformations is, in this case, readily approximated, since each series is alternating. If we consider the final approximants for the successive series $S, T, T^{\prime}, T^{\prime \prime}$, and $T^{\prime \prime \prime}$ we obtain the sequence: $0.7456349206 ; \quad 0.6933473389 ; \quad 0.6931508286$; $0.6931473540 ; 0.6931471961$; the last value being correct to better than 2 in the eighth place. This is not an infinite sequence, since $T^{\prime \prime \prime}$ has too few terms to permit continuation of the transformation. If, however, we assume it represents the first terms of an infinite sequence converging to $\log 2$, without proof other than appearance, we get the series

$$
\begin{aligned}
& U=.7456349206-.0522875817-.0001965103 \\
& -.0000034746-.0000001580-\ldots .
\end{aligned}
$$

Assuming further that this series is of sufficient smoothness to justify application of the $T$ transformation, we get

$$
\begin{aligned}
U^{\prime}= & .6931500873-.0000027958 \\
& -.0000001029-\cdots \\
U^{\prime \prime}= & 0.6931471846 .
\end{aligned}
$$

The $U$ series final approximants form the sequence:

$$
0.6931471961 ; 0.6931471885 ; 0.6931471846 \text {. }
$$

Assuming these also are initial terms of an infinite sequence converging to $\log 2$, we write

$V=0.6931471961-0.0000000075$ $-0.0000000039-\ldots$.

which transforms into

$$
V^{\prime}=0.6931471804 .
$$


The heuristic process stops at this point because of lack of terms, but this last value is remarkably close to the correct value to 10 places of

$$
\log 2=0.6931471806 \text {. }
$$

In fact, because only 10 digits were carriad throughout the calculations, the agreement is about 1 place better than could be expected even were the later steps proved fully justifiable. Note that, by this method, we have achieved an accuracy using 9 terms of the original series, which is better than that obtained by summing directly hundreds of millions of additional terms. The effect of the additional terms is included by considerations of "smoothness".

We could also have applied the $W$ transformation, since the hypothesis of theorem 12 is satisfied. In this case, the successive transformed series are:

$$
\begin{aligned}
W & =.6875000000+.0069444444-.0017361111 \\
& +.0006250000-.0002777778+.0001417234 \\
& -.0000797194+\ldots . \\
W^{\prime} & =.6931399632+.0000089884-.0000022983 \\
& +.0000007115-\ldots . \\
W^{\prime \prime} & =.6931471793+\ldots .
\end{aligned}
$$

omitting steps that involve terms not "smoothly" related to the remainder because of differences in form indicated by (19). The final approximants for series $S, W, W^{\prime}$, and $W^{\prime \prime}$ are

\subsection{6; $0.6931175595 ; 0.6931473648$;}

0.6931471794 .

Assuming these to be the first terms of an infinite sequence converging to $\log 2$, we get the series

$$
\begin{aligned}
& X=.7456349206-.0525173612-.0000298053 \\
& -.0000001854-\ldots .
\end{aligned}
$$

Applying the $W$ transformation to this, we get

$$
X^{\prime}=0.6931471806 \text {. }
$$

We thus see, as might have been expected, that the $W$ transformation is more effective than the $T$ transformation. However, the simpler calculations in the case of the $T$ transformation probably more than offset the additional number of steps required as compared to the $W$ transformation.

\section{Example 2:}

$$
S=\frac{1}{2}-1+\frac{1}{4}-\frac{1}{3}+\frac{1}{6}-\frac{1}{5}+\frac{1}{8}-\frac{1}{7}+\ldots .
$$

After seeing how advantageous the transformations are when "smoothness" exists, we choose this example to show the ill effects of irregularity. This is, as may be seen by examiration, identical with the series used in example 1 except that the sign is reversed and odd and even terms are interchanged. In spite of being only conditionally convergent this interchange does not affect the sum, which is $-\log 2$. If the type of irregularity is noted, the best procedure would be to remove it by rearranging the series (in this case obtaining the form given in example 1), by combining terms (in this case obtaining the form given in example 5), or by splitting it into several individually "smooth" series (not possible in this case since this leads to two properly divergent series). Suppose, however, we do not note these possibilities but attempt to work with the series as it stands. It is obvious that theorems 11 and 12 are inapplicable. So is theorem 5. However, theorem 2 applies except that $1+R_{n} \rightarrow 0$, and hence we have no statement about relative speed of convergence, although we know that the $T$ transformation leads to a convergent series. In fact, we find that

$$
\begin{aligned}
& T=.16667-.46667-.09286-.07937 \\
& -.03535-.03217-.01859-.01737 \\
& -.01146-. . .
\end{aligned}
$$

The terms are certainly smaller than those of $S$. However, the character of the series is changed from alternating to terms having the same sign, and this makes the error for a given number of terms of the same order as that of the original series, being approximately the average of the errors found for the original series for consecutive partial sums. We have thus not gained by the use of the transformation. Examination of $T$ shows that $R$ exists and has the value 1 and that $n\left(1-b_{n} / b_{n-1}\right) \rightarrow \frac{7}{2}$ for $n$ even and $\rightarrow \frac{1}{2}$ for $n$ odd, so that $T$ satisfies theorem 6 and can itself be transformed into a new convergent series $T^{\prime}$. Of course, even at this step, if the type of peculiarity were noted, it would be possible to combine pairs of terms or to separate the odd and even terms into separate series to be separately summed, obtaining "smooth" series in either case that would satisfy the hypothesis of more useful theorems. If, however, we continue with the transformation to $T^{\prime}$, we may note that, although it converges, its terms are larger than those of $T$ since, from the given limits for $n\left(1-b_{n} / b_{n-1}\right)$ we have $\left|P_{n}^{\prime}\right| \sim \frac{12}{7} n \rightarrow \infty$. In fact, $T^{\prime}$ is quite similar in convergence and form to $S$ with terms alternating in sign and tending to $\frac{12}{7}$ those of $S$ for large $n$. The $T^{\prime}$ series is

$T^{\prime}=.41592-.52315-.40310-.32859-.28078-.$. 
We may now attempt to proceed in accord with the remark following theorem 8 to get the equivalent and, we hope, more rapidly converging series

$$
V=-\frac{7}{5}\left(T^{\prime}-\frac{12}{7} S\right)
$$

which is, numerically,

$$
V=1.78229-1.66759-.03566-.33997-.00693
$$

This series, unfortunately, is even worse than $T$. The irregularity is much more pronounced.

We may try the $W$ transformation instead. It is easy to show that its resulting series is convergent. It is

$$
\begin{aligned}
W= & -.12500-.22917-.08333-.05417-.03333 \\
& -.02470-.01786 .-. .
\end{aligned}
$$

and is certainly no more rapidly convergent than the $T$ series. If we transform this again, we obtain

$$
W^{\prime}=.10417-.66477+.39964-.44015+\ldots,
$$

which does not appear promising.

We may thus conclude that, if a certain amount of smoothness is lacking in the original series, the transformations, while leading to convergent series, may not help in evaluation but may help in indicating the character of the irregularity and thus suggest some method of modifying the original series or one of its transformations, by grouping, rearranging, or splitting into subseries.

Example 3:

$$
\begin{aligned}
S= & 1+0-\frac{1}{2}+0+0+\frac{1}{4}+ \\
& 0+0+0-\frac{1}{8}+0+0+0+0+\frac{1}{16}+\ldots .
\end{aligned}
$$

This example shows the effect of a somewhat different type of irregularity. Here we readily find that $T$ and $W$ are identical with $S$ for any consistent interpretation of formulae, and hence the schemes have no value.

Example 4:

$$
S=1+\frac{1}{2}-\frac{1}{4}-\frac{1}{8}+\frac{1}{16}+\frac{1}{32}-\frac{1}{64}-\frac{1}{128}+\ldots .
$$

This is still another example of irregularity. Here we readily find that

$$
T=2-\frac{2}{3}\left(1+\frac{1}{2}-\frac{1}{4}-\frac{1}{8}+\frac{1}{16}+\frac{1}{32}-\frac{1}{64}-\frac{1}{128}+\ldots\right),
$$

which certainly has the same type of convergence as $S$. However, we may now utilize theorem 1, which states that $T=S$ to obtain $S=2-\frac{2}{3} S$ from which we get the sum $S=\frac{6}{5}$. This particular series can, of course, be summed more easily by grouping pairs of terms to give

$$
S=1+\frac{1}{4}-\frac{1}{16}+\frac{1}{64}-\ldots,
$$

which is, except for the first term, a simple geometric series converging to $\frac{6}{5}$.

The $W$ transformation, in this case, gives

$$
W=\frac{10}{7}+\frac{4}{7}-\frac{6}{7}-\frac{1}{7}+\frac{3}{14}+\frac{1}{28}-\ldots .
$$

and offers no apparent advantages.

\section{Example 5:}

$S=\frac{1}{1.2}-\frac{1}{2.4}-\frac{1}{3.8}+\frac{1}{4.16}+\frac{1}{5.32}-\frac{1}{6.64}-\frac{1}{7.128}+\cdots$

This is a somewhat less obvious application of the remark following theorem 8 .

We readily find that

$$
\begin{aligned}
T= & \frac{2}{5}-\frac{21}{10.3 .8}+\frac{68}{33.4 .16}+\frac{155}{66.5 .32}- \\
& \frac{196}{85.6 .64}-\frac{497}{204.7 .128}+\cdots,
\end{aligned}
$$

and that terms tend to $\frac{8}{3}$ of the next to corresponding term in $S$. We thus take, as a more convergent series with the same sum $V=\frac{3}{5}\left(\frac{8 S}{3}-T\right)$ with the two series displaced one term prior to combining, thus obtaining

$$
V=\frac{9}{25}-\frac{17}{1200}+\frac{1}{176}+\frac{21}{17600}-\frac{23}{40800}-\frac{47}{304640}+\cdots,
$$

which converges more rapidly than the initial series. A preferable procedure is, of course, to combine pairs of terms.

Example 6:

$$
S=\frac{1}{1.2}+\frac{1}{3.4}+\frac{1}{5.6}+\frac{1}{7.8}+\frac{1}{9.10}+\cdots .
$$

This is the same series as used in example 1 but with pairs of terms added. It is readily seen that this series satisfies the hypothesis of theorem 8 with $Q=2$. We may, thus, proceed as there indicated to get the successive more rapidly converging series:

$$
\begin{aligned}
V= & \frac{7}{10}-\frac{1}{180}-\frac{1}{1170}-\frac{3}{12376}-\cdots- \\
& \frac{3}{2 n(2 n-1)(4 n-3)(4 n+1)}-\cdots,
\end{aligned}
$$


for which $Q=4$,

and

$$
V^{\prime}=\frac{4117}{5940}+\frac{49}{1196910}+\cdots \cdot
$$

which, as far as we have gone, gives the value 0.693139 , which is correct to 8 in the last digit. Here, also, if desired, we may follow Example 1 in assuming that the last partial sums of $S, V$, and $V^{\prime}$ form the first terms of a reasonably smooth infinite series and thus improve results somewhat further with the given number of terms.

We also note that theorem 9 is applicable. If we use the $W$ transformation, we get

$$
W=\frac{11}{16}+\frac{1}{240}+\frac{1}{1120}+\cdots,
$$

which, so far as we have gone, gives the value 0.69306 , which is in error about 8 in the last digit.

Example \%:

$$
S=1+\frac{1}{2}-\frac{1}{3}-\frac{1}{4}+\frac{1}{5}+\frac{1}{6}-\frac{1}{7}-\frac{1}{8}+\frac{1}{9}+\cdots
$$

This example is included to show that there exist convergent series, even with a certain type of smoothness, for which the $T$ transformation leads to a divergent series. Here $T$ is given by

$$
T=2-\frac{7}{10}-\frac{17}{15}+\frac{31}{36}+\frac{49}{45}-\frac{71}{78}-\frac{97}{91}+\frac{127}{136}+\cdots,
$$

and terms tend toward \pm 1 .

The $W$ transformation does, however, lead to a convergent series albeit no more rapidly so than the original. The resulting series is

$$
W=\frac{17}{12}+\frac{1}{6}-\frac{77}{120}-\frac{1}{20}+\frac{319}{840}+\frac{1}{42}-\frac{275}{1008}-\cdots
$$

Example 8:

$$
S=1+\frac{1}{1+1}+\frac{1}{2^{2}}+\frac{2^{2}}{2^{4}+1}+\frac{1}{3^{2}}+\frac{3^{2}}{3^{4}+1}+\cdots
$$

This is another example showing that there exist convergent series, even with sufficient smoothness to have $R$ exist $=1$, which lead to divergent series upon application of the $T$ transformation. Here

$$
0<S_{2 n-1}<S_{2 n}<2 \sum_{1}^{n+1} \frac{1}{2 n^{2}},
$$

so that $S$ converges. However,

so that

$$
T_{2 n}=S_{2 n}+(n+1)^{2}>(n+1)^{2} \rightarrow \infty .
$$

The $W$ transformation leads, in this case, to a convergent series since, as may be readily verified,

and

$$
\frac{a_{2 n}\left(1-R_{2 n+1}\right)}{1-2 R_{2 n+1}+R_{2 n} R_{2 n+1}} \sim \frac{1}{n^{2}} \rightarrow 0
$$

$$
\frac{a_{2 n-1}\left(1-R_{2 n}\right)}{1-2 R_{2 n}+R_{2 n} R_{2 n-1}} \sim-\frac{1}{2 n^{5}} \rightarrow 0 .
$$

Example 9:

$$
\begin{aligned}
S= & 1+\frac{1}{2^{2}}+\frac{2^{3}-2}{2^{2} \cdot 3.4}+\frac{1}{2^{2}}+\frac{1}{3^{2}}+ \\
& \frac{3^{3}-2}{2.3^{2} \cdot 4.5}+\frac{1}{3^{2}}+\frac{1}{4^{2}}+\frac{4^{3}-2}{3.4^{2} .5 .6}+\frac{1}{4^{2}}-\ldots .
\end{aligned}
$$

This is an example showing that there exist convergent series, even with sufficient smoothness to have $R$ exist and have the value 1 , which lead to divergent series upon application of the $W$ transformation and this despite the fact that the $\mathrm{T}$ transformation leads to convergent series. Here $0<S_{3 n-2}<S_{3 n-1}<S_{3 n}<3 \sum_{1}^{n+1} \frac{1}{n^{2}}$ so that $S$ converges. However,

$$
\frac{a_{3 n+1}\left(1-R_{3 n+2}\right)}{1-2 R_{3 n+2}+R_{3 n+1} R_{3 n+2}}=\frac{n+2}{2} .
$$

so that

$$
W_{3 n+1}=S_{3 n+1}+\frac{n+2}{2}>\frac{n+2}{2} \rightarrow \infty .
$$

Furthermore,

$$
\begin{aligned}
& \frac{a_{3 n-1}}{1-R_{3 n-1}}=\frac{(n+1)^{3}-2}{(n+1)^{3}(2 n+1)} \rightarrow 0 . \\
& \frac{a_{3 n}}{1-R_{3 n}}=-\frac{(n+1)^{3}-2}{(n+1)^{3}(2 n+1)} \rightarrow 0 .
\end{aligned}
$$

and

$$
\frac{a_{3 n+1}}{1-R_{3 n+1}}=\frac{1}{2 n+3} \rightarrow 0
$$

so that

$$
T_{n} \sim S_{n} \rightarrow S \text {. }
$$

\section{Original Series Divergent}

General: There has been considerable study made of methods of assigning meanings to Divergent Series. One possible way to assign such a meaning to a Divergent Series $S$ is to consider it as the value of the function $S(x)$ at $x=1$, where $S(x)$ is defined for sufficiently small $x$ by the power series $\sum_{0}^{\infty} a_{n} x^{n}$ and for larger $x$ by analytic continuation or limit processes. If $R_{n}$ remains bounded as $n \rightarrow \infty$, there will 
always be a region in which the power series converges (certainly when $|x|$ is less than the lower bound of $1 /$ $\left|R_{n}\right|$ ). If, however, $R_{n}$ is unbounded, the power series may converge only at the origin and does not, therefore, define an analytic function over any region. In any case, it may not be possible to find an analytic continuation or limit for $x=1$. Another method, developed by Borel, is to consider the power series $S_{B}(x)=\sum_{0}^{\infty} \frac{a_{n} x^{n}}{n !}$ as defining some function of $x$ and then to define the sum of the divergent series $S$ by the integral

$$
S=\int_{0}^{\infty} e^{-x} S_{B}(x) d x
$$

Still another way of treating the matter is to consider the power series $S(x)$, even if it diverges for all $x$ no matter how small, as an asymptotic representation of a function $F(1 / x)$. Then, if $F(1 / x)$ is defined at $x=1$, we may take $S=F(1)$. Since, in general, any asymptotic series may represent an infinity of functions, this method requires some additional limitations in regard to the behavior of $F\left(\frac{1}{x}\right)$ to insure uniqueness.

The use of the transformations discussed in this paper may, in some cases, lead to "equivalent" convergent series and thus to another definition of sum of divergent series. In many cases, however, the transformation merely serves to obtain an equivalent series, also divergent, but diverging more slowly. The new series may be easier to sum than the old. In particular, if the original series can be considered as the result of substituting a particular value for the variable in an asymptotic series, the resulting series may be similarly regarded but with individual terms smaller than for the original so that, if the error stopping at any term is limited by the magnitude of the next term, the transformed series yields a more precise sum. The theory is, obviously, more difficult by far than for convergent series and has not been developed beyond a rudimentary stage by the author. Heuristic application to particular examples does, however, indicate considerable power in this method.

Theorem 13. If there is a definition $M$ for the sum of an infinite series that has the following properties:

A. If $\sum_{0}^{\infty} c_{n}$ is summable $M$ with sum $C$ and $\sum_{0}^{\infty} d_{n}$ is summable $M$ with sum $D$, then $\sum_{0}^{\infty}\left(c_{n} \pm d_{n}\right)$ is summable $M$ with sum $C \pm D$.

B. If $\sum_{0}^{\infty} c_{n}$ is summable $M$ with sum $C$ then $\sum_{0}^{\infty} c_{n}$ is summable $M$ with sum $C+c_{0}$, and if both $S$ and $V=\sum_{0}^{\infty} a_{n} /\left(1-R_{n}\right)$ are summable $M$, then $T$ is summable $M$ with the same sum as $S$.

Proof: By condition B, we have

$$
\operatorname{Sum}_{M}\left[0+\sum_{1}^{\infty} \frac{a_{n}}{1-R_{n}}\right]=\operatorname{Sum}_{M} V \text {. }
$$

Hence, by condition A,

$$
\operatorname{Sum}_{M}\left[\frac{a_{1}}{1-R_{1}}+\sum_{1}^{\infty}\left(\frac{a_{n+1}}{1-R_{n+1}}-\frac{a_{n}}{1-R_{n}}\right)\right]=0
$$

and

$\operatorname{Sum}_{M}\left[a_{0}+\frac{a_{1}}{1-R_{1}}+\sum_{1}^{\infty}\left(a_{n}+\frac{a_{n+1}}{1-R_{n+1}}-\frac{a_{n}}{1-R_{n}}\right)\right]=\operatorname{Sum}_{M} S$.

But, from (9),

and

$$
b_{0}=a_{0}+\frac{a_{1}}{1-R_{1}}
$$

$$
b_{n}=a_{n}+\frac{a_{n+1}}{1-R_{n+1}}-\frac{a_{n}}{1-R_{n}}, n>0 .
$$

Hence,

$$
\operatorname{Sum}_{M} T=\operatorname{Sum}_{M} S .
$$

Remarks. The definitions that satisfy the conditions of this theorem include Cesào and Hölder summability of any order and absolute Borel summability. Condition B can be modified to read: "If $\sum_{1}^{\infty} c_{n}$ is summable $M$ with sum $C$ then, if $\sum_{0}^{\infty} c_{n}$ is summable $M$, its sum is $C+c_{0}$ ", provided we add to the hypothesis: "and $V^{\prime}=\left[0+\sum_{1}^{\infty} \frac{a_{n}}{1-R_{n}}\right]$ is summable M." The modified condition is met by Borel summability without the requirement that it be absolute. ${ }^{12}$

Theorem 14: If $S$ is such that $\left|R_{n}\right| \leq M$ for all $n$ and if $T$ converges absolutely, then $T$ is the limit as $z \rightarrow 1$ of the analytic continuation of $S(z)=\sum_{0}^{\infty} a_{n} z^{n}$.

Proof: Consider the series

$$
\begin{aligned}
T(z) & =\sum_{0}^{\infty} a_{n}\left(\frac{1}{1-R_{n+1} z}-\frac{1}{1-R_{n} z}\right) z^{n} \\
& =\sum_{0}^{\infty} \frac{\left(1-R_{n+1}\right)\left(1-R_{n}\right)}{\left(1-R_{n+1} z\right)\left(1-R_{n} z\right)} b_{n} z^{n+1}
\end{aligned}
$$

and the region bounded by the circular arc $|z|=1$ and the line segments $x=0, y=-1 / 2, x=1 / 2 M$, and $y=1-x$. If $M<1$, then $S$ converges and, by Theorem 1, we have $T=S=S(1)$ satisfying the present theorem in trivial fashion. If $M \geq 1$, $1 / 2 M \leq 1 / 2$ and the region is as indicated in the diagram on p. 242 . Throughout the region and its boundary, we have $|z| \leq 1$. In the left-hand portion, $0 \leq x \leq 1 / 2 M$ so that

$$
\left|1-R_{n} z\right| \geq\left|1-R_{n} x\right| \geq 1-\left|R_{n}\right||x| \geq 1 / 2
$$

while

$$
\left|1-R_{n}\right| \leq 1+\left|R_{n}\right| \leq M+1 .
$$

${ }^{12}$ W. B. Ford, Studies on divergent series and summability, p. 89 (Macmillan, New York, N. Y., 1916). 


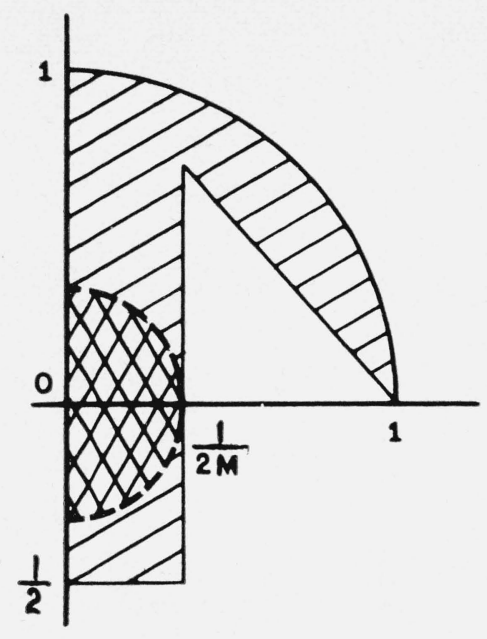

Hence

$$
\left|1-R_{n}\right| /\left|1-R_{n} z\right| \leq 2(M+1) .
$$

In the right-hand portion, we have $y \geq 1-x$ so that

$$
\begin{aligned}
\left|1-R_{n} z\right| & =\sqrt{\left(1-R_{n} x\right)^{2}+R_{n}^{2} y^{2}} \\
& \geq \sqrt{\left(1-R_{n} x\right)^{2}+R_{n}^{2}(1-x)^{2}} \\
& =\sqrt{\left(1-R_{n}\right)^{2}+\left(1+R_{n}-2 R_{n} x\right)^{2}} / \sqrt{2} \\
& \geq\left|1-R_{n}\right| / \sqrt{2} .
\end{aligned}
$$

Hence

$$
\left|1-R_{n}\right| /\left|1-R_{n} z\right| \leq \sqrt{2}<2(M+1) .
$$

Therefore, throughout the region and its boundary,

where

$$
\left|f_{n}(z)\right|<4(M+1)^{2}\left|b_{n}\right|
$$

$$
f_{n}(z)=\frac{\left(1-R_{n+1}\right)\left(1-R_{n}\right) b_{n} z^{n+1}}{\left(1-R_{n+1} z\right)\left(1-R_{n} z\right)} .
$$

But, by hypothesis, $\sum_{0}^{\infty}\left|b_{n}\right|$ is convergent. Hence $T(z)$ is uniformly convergent throughout the region and its boundary. ${ }^{13}$ Moreover, $f_{n}(z)$ is a rational function of $z$ and is therefore analytic except at the points $z=1 / R_{n}$ and $z=1 / R_{n+1}$, which are poles. Since convergence of $T$ reverses finiteness of the $b_{n}$, we cannot have $R_{n}=1$ for any $n$. Also, by hypothesis, $\left|R_{n}=\right| \leq M$. Hence these poles do not fall within the region or on its boundary, and $f_{n}(z)$ is analytic within the region and continuous throughout the region and its boundary. Hence $T(z)$ is analytic within the region, ${ }^{14}$ and is continuous throughout the region and its boundary. ${ }^{15}$ Thus the limit of $T(z)$ as $z \rightarrow 1$ is $T(1)=T$. Also, $S(z)$ is a power series with radius of convergence at least as great as $1 / 2 M$, since $S(1 / 2 M)$ converges, having the ratio of each term to its predecessor less than or equal to $1 / 2$.

\footnotetext{
13 Whittaker and Watson, Modern analysis, p. 49 (Cambridge University Press, 1915)

14 Modern analysis, p. 91

15 Modern analysis, p. 47.
}

Hence $S(z)$ is analytic in the double-hatched region of the diagram bounded by the semicircle $(z)=1 / 2 M$ and the $y$ axis and, in particular, converges for $z=x$ for any $x$ in the range $0<x<1 / 2 M . T(z)$ is also analytic in the same region since it is included in the region previously considered and converges for all points on the portion of the $x$ axis included. Hence by theorem 1, $T(z)$ is identical with $S(z)$ in this region and is therefore an analytic continuation of $S(z)$ throughout the larger region.

Theorem 15. If $S$ diverges, $R_{n}>0$ for $n$ sufficiently large, and there are an infinity of values of $n$ for which $R_{n} \leq 1$, then $T$ diverges.

Proof: Since $S$ diverges, we cannot have $a_{n}=0$ for all $n$ sufficiently large. Hence, if there are an infinity of values of $n$ for which $a_{n}=0$, there must be an infinity of such values for which $a_{n-1} \neq 0$, and hence $R_{n}=0$ contrary to hypothesis. If there are an infinity of values of $n$ for which $R_{n}=1$ but $a_{n} \neq 0$, we have each corresponding $T_{n-1}$ infinite and thus $T$ diverges. Finally, if there are an infinity of values of $n$ for which $R_{n}<1$, then, for each of these $n, a_{n} /\left(1-R_{n}\right)$ has the same sign as $a_{n}$, and hence as $S_{n-1}$ for $n$ sufficiently large so that $\left|T_{n-1}\right|>\left|S_{n-1}\right|$ for an infinity of values of $n$ and thus is unbounded.

Theorem 16. If $S$ diverges, $R_{n}>0$ for $n$ sufficiently large, and there is an infinite sequence of values of $n$, say $n_{1}<n_{2}<n_{3}<\ldots$ such that $R_{n_{i}} \rightarrow 1$ as $i \rightarrow \infty$, then $T$ diverges.

Proof: In view of the proof of theorem 15, we may limit attention to $a_{n} \neq 0$ and $R_{n}>1$ for $n$ sufficiently large. Since the $a_{n}$ are all of like sign for $n$ sufficiently large, we may take them as positive for convenience, an almost identical proof applying if they are negative. Let, therefore, $N_{0}$ be such that, for $n \geq N_{0}, a_{n}>0$ and $R_{n}>1$. Since $R_{n_{i}} \rightarrow 1$, there exists a value $N \geq N_{0}$ for which $R_{N}<R_{N_{0}}$. Let $N$, be the least such value. Thən, for every $n$ such that $N_{0} \leq n<N_{1}$, we must have $R_{n} \geq R_{N_{0}}>R_{N_{1}}$. Starting with $N_{1}$, we may similarly get a value $N_{2}$ such that, for $N_{1} \leq n<N_{2}$ we have, $R_{n} \geq R_{N_{1}}>R_{N_{2}}$. Combining with the preceding, we then have $R_{n}>R_{N_{2}}$ for all $n$ such that $N_{0} \leq n<N_{2}$. Continuing in similar fashion, we get an infinite sequence $N$ with the property that $R_{n}>R_{N_{j}}$ for all $n$ in the range $N_{0} \leq n<N_{j}$ and with $N_{j}>N_{j-1}$. Now, from (5), we have

$$
\begin{aligned}
T_{\left(N_{i}-1\right)}= & S_{N_{0}}+a_{\left(N_{0}+1\right)}+a_{\left(N_{0}+2\right)}+\ldots+a_{\left(N_{i}-2\right)}- \\
& a_{\left(N_{j}-1\right)} /\left(R_{N_{j}}-1\right) \\
= & S_{N_{0}}-\left\{a_{\left(N_{0}+1\right)}+\left[a_{\left(N_{0}+2\right)}-R_{N_{i}} a_{\left(N_{0}+1\right)}\right]+\right. \\
& {\left[a_{\left(N_{0}+3\right)}-R_{N_{j}} a_{\left(N_{0}+2\right)}\right]+\ldots+\left[a_{\left(N_{i}-1\right)}-\right.} \\
& \left.\left.R_{N_{j}} a_{\left(N_{j}-2\right)}\right]\right\} /\left(R_{N_{i}}-1\right) .
\end{aligned}
$$

But $a_{n}=R_{n} a_{n-1}>R_{N_{j}} a_{n-1}$ for $N_{0} \leq n<N_{j}$. Hence all terms in the braces are positive and 


$$
T_{\left(N_{j}-1\right)}<S_{N_{0}}-a_{\left(N_{0}+1\right)} /\left(R_{N_{j}}-1\right)
$$

But $R_{n_{i}}>R_{N_{i}}>1$ for $N_{0} \leq n_{i}<N_{j}$. Hence $R_{N_{j}}-1$ $\rightarrow 0$ since $R_{n_{i}} \rightarrow 1$ and thus $T_{\left(N_{j}-1\right)} \rightarrow-\infty$, so that $T$ diverges.

Theorem 1\%. If $S$ is a divergent series and

$$
R_{n}=-1+\frac{\alpha 1}{n}+\frac{\alpha 2}{n^{2}}+\frac{\alpha 3}{n^{3}}+\ldots
$$

is valid for all sufficiently large $n$, then the repeated application of the $T$ transformation to $S$ and to each derived series in turn will, after $m$ applications, yield a series $T^{(m)}$ which converges, where $m$ is the smallest integer exceeding $-\alpha_{1} / 2$. Moreover, $T^{(m)}=\lim _{x \rightarrow 1-0} \sum_{0}^{\infty} a_{n} x^{n}$.

Proof: We note that $\alpha_{1} \leq 0$ since, otherwise, $\left|R_{n}\right|<1$ for $n$ sufficiently large so that $S$ is an alternating series with terms steadily decreasing in magnitude and converges contrary to hypothesis. Using the analysis of theorem 11 in so far as it does not depend upon convergence of $S$, we see that $T$ terminates if all the $\alpha_{i}=0$ and that, otherwise, $T$ is an infinite series with ratio of each term to its predecessor given by

$$
\frac{b_{n}}{b_{n-1}}=-1+\frac{\beta_{1}}{n}+\frac{\beta_{2}}{n^{2}}+\frac{\beta_{3}}{n^{3}}+\cdots
$$

where $\beta_{1}=\alpha_{1}+2$. If $\alpha_{1}>-2$, then $\alpha_{1}+2>0$ and $T$ converges since, for $n$ sufficiently large, it is an alternating series with terms of steadily decreasing magnitude. If $\alpha_{1}+2=0, T$ will converge if the first $\beta_{i}$ that is not zero is negative; it will diverge if all the $\beta_{i}$ are zero or if $\alpha_{1}<-2$. When $T$ diverges, it satisfies the same conditions as the hypothesis prescribed for $S$. If we repeat the same transformation on $T$ to get a series $T^{(2)}=\sum_{0}^{\infty} b_{n}^{(2)}$ then either $T^{(2)}$ terminates or is an infinite series with ratio of each term to its predecessor given by

$$
\frac{b_{n}^{(2)}}{b_{n-1}^{(2)}}=-1+\frac{\beta_{1}^{(2)}}{n}+\frac{\beta_{2}^{(2)}}{x^{2}}+\frac{\beta_{3}^{(2)}}{n^{3}}+\cdots,
$$

where $\beta_{1}^{(2)}=\beta_{1}+2=\alpha_{1}+4$. By simple induction, we thus see that, for $-\alpha_{i}$ not an even integer, it will take $m$ successive transformations to obtain a series $T^{(m)}=\sum_{0}^{\infty} b_{n}^{(m)}$ with magnitude of terms decreasing for terms sufficiently far out (that is with $\beta_{1}^{(m)}>0$ ) so that $T^{(m)}$ will then converge. If $-\alpha_{1}$ is an even integer, we get $\beta_{1}^{(m-1)}=0$, and it is possible for $T^{(m-1)}$ to converge. In view of theorem $2, T^{(m)}$ will also converge in this case.

As concerns the values of $T^{(m)}$, we see from Lemma
4 below, that $\lim _{x \rightarrow 1-0} \sum_{0}^{\infty} a_{n} x^{n}$ and $\lim _{x \rightarrow 1-0} \sum_{0}^{\infty} a_{n} x^{n} /\left(1-R_{n}\right)$ both exist. Now the limit operation stated is a definition of summability that satisfies the conditions of theorem 13. Hence $\lim _{x \rightarrow 1-0} \sum_{0}^{\infty} b_{n} x^{n}=\lim _{x \rightarrow 1-0} \sum_{0}^{\infty} a_{n} x^{n}$. Since $T$ also satisfies the conditions of the lemma, we similarly get

$\lim _{x \rightarrow 1-0} \sum_{0}^{\infty} b_{n}{ }^{(2)} x^{n}=\lim _{x \rightarrow 1-0} \sum_{0}^{\infty} b_{n} x^{n}$ so that, by induction, $\lim _{x \rightarrow 1-0} \sum_{0}^{\infty} b_{n}{ }^{(m)} x^{n}=\lim _{x \rightarrow 1-0} \sum_{0}^{\infty} a_{n} x^{n}$. Since $T^{(m)}$ converges, $\lim _{x \rightarrow 1-0} \sum_{0}^{\infty} b_{n}{ }^{(m)} x^{n}=T^{(m)}$ completing the proof.

\section{Lemma 4. If S has terms such that}

$$
R_{n}=-1+\frac{\alpha_{1}}{n}+\frac{\alpha_{2}}{n^{2}}+\frac{\alpha_{3}}{n^{3}}+\cdots
$$

is valid for all sufficiently large $n$, then $\lim _{x \rightarrow 1-0} \sum_{0}^{\infty} a_{n} x^{n}$ and $\lim _{x \rightarrow 1-0} \sum_{0}^{\infty} a_{n} x^{n} /\left(1-R_{n}\right)$ both exist.

Proof: Let $F(x)=(1+x)^{k} \sum_{0}^{\infty} a_{n} x^{n}$ where $k>1-\alpha_{1}$ is an integer. Then $F(x)=P(x)+f(x)$ where $P(x)$ is a polynomial of degree $k-1$ in $x$ and

$$
\begin{aligned}
f(x)= & \sum_{0}^{\infty} a_{n} x^{n+k k}\left[1+k R_{n+1}+\frac{k(k-1)}{2} R_{n+1} R_{n+2}+\right. \\
& \frac{k(k-1)(k-2)}{3 !} R_{n+1} R_{n+2} R_{n+3} \ldots+ \\
& \left.R_{n+1} R_{n+2} R_{n+3} \ldots R_{n+k}\right] .
\end{aligned}
$$

Let us designate the quantity in the brackets as $A_{k}(n)$. Then

$$
\begin{aligned}
A_{1}(n) & =1+R_{n+1}=\frac{\alpha_{1}}{n+1}+\frac{\alpha_{2}}{(n+1)^{2}}+\frac{\alpha_{3}}{(n+1)^{3}}+\ldots \\
& =\frac{\alpha_{1}}{n}+\frac{\left(\alpha_{2}-\alpha_{1}\right)}{n^{2}}+\ldots,
\end{aligned}
$$

also if

$$
\begin{aligned}
A_{k}(n)= & \frac{\alpha_{1}\left(\alpha_{1}+1\right)\left(\alpha_{1}+2\right) \ldots\left(\alpha_{1}+k-1\right)}{n^{k}}+ \\
& \frac{\delta_{1}(k)}{n^{k+1}}+\frac{\delta_{2}(k)}{n^{k+2}}+\cdots,
\end{aligned}
$$

then

$$
\begin{aligned}
A_{k}(n+1)= & \frac{\alpha_{1}\left(\alpha_{1}+1\right)\left(\alpha_{1}+2\right) \ldots \cdot\left(\alpha_{1}+k-1\right)}{(n+1)^{k}}+ \\
& \frac{\delta_{1}(k)}{(n+1)^{k+1}}+\frac{\delta_{2}(k)}{(n+1)^{k+2}}+\ldots
\end{aligned}
$$

so that 


$$
\begin{aligned}
R_{n+1} A_{k}(n+1) & =-\frac{\alpha_{1}\left(\alpha_{1}+1\right)\left(\alpha_{1}+2\right) \ldots\left(\alpha_{1}+k-1\right)}{(n+1)^{k}}+\frac{\alpha_{1}^{2}\left(\alpha_{1}+1\right)\left(\alpha_{1}+2\right) \ldots\left(\alpha_{1}+k-1\right)-\delta_{1}(k)}{(n+1)^{k+1}}+\ldots \\
& =-\frac{\alpha_{1}\left(\alpha_{1}+1\right)\left(\alpha_{1}+2\right) \ldots\left(\alpha_{1}+k-1\right)}{n^{k}}+\frac{\alpha_{1}\left(\alpha_{1}+1\right)\left(\sigma_{1}+2\right) \ldots\left(\alpha_{1}+k-1\right)\left(\alpha_{1}+k\right)-\delta_{1}(k)}{n^{k+1}}+\cdots
\end{aligned}
$$

and

$$
A_{k+1}(n)=A_{k}(n)+R_{n+1} A_{k}(n+1)=\frac{\alpha_{1}\left(\alpha_{1}+1\right)\left(\alpha_{1}+2\right) \cdots\left(\alpha_{1}+k\right)}{n^{k+1}}+\frac{\delta_{1}(k+1)}{n^{k+2}}+\cdots
$$

Hence we have established the basis for an induction proving that the form assumed is correct. Thus, for $n$ sufficiently large, we have $\left|A_{k}(n)\right|<\mid \alpha_{1}+k^{k}{ }^{k} / n^{k}$ and, for $|x| \leq 1$, each term in $f(x)$ is less in absolute value than the corresponding term in $f=\sum_{0}^{\infty}\left|\alpha_{1}+k\right|^{k}$ $\times\left|a_{n}\right| / n^{k}$. But the ratio of each term of the latter to its predecessor is given by $\left|R_{n}\right|(1-1 / n)^{k}=1-$ $\left(k+\alpha_{1}\right) / n+$ terms of higher degree in $1 / n$ and $k$ was chosen greater than $1-\alpha_{1}$ so that $k+\alpha_{1}>1$. Hence $f$ converges and, hence, $f(x)$ converges for $|x| \leq 1$. Also, since $P(x)$ is a polynomial, it is analytic for all $x$. Hence $F(x)$ is analytic at least for $|x| \leq 1$ and we have $\sum_{0}^{\infty} a_{n} x^{n}$ convergent and equal to $F(x) /(1+x)^{k}$ for all $|x|<1$ so $\lim _{x \rightarrow 1-0} \sum_{0}^{\infty} a_{n} x^{n}$ exists; its value is $F(1) / 2^{k}$.

Moreover, the ratio of each term of $\sum_{0}^{\infty} a_{n} /\left(1-R_{n}\right)$ to its predecessor is, for $n$ sufficiently large,

$$
\frac{R_{n}\left(1-R_{n-1}\right)}{1-R_{n}}=-1+\frac{\gamma_{1}}{n}+\frac{\gamma_{2}}{n^{2}}+\frac{\gamma_{3}}{n^{3}}+\ldots
$$

where $\gamma_{1}=\alpha_{1}$ as may be shown by an analysis similar to that used in the proof of theorem 11. Hence this series satisfies the hypothesis in the same way as $S$ and, by similar reasoning, $\lim _{x \rightarrow 1-0} \sum_{0}^{\infty} a_{n} x^{n} /\left(1-R_{n}\right)$ exists.

Example 10: $S=1-1+1-1+1-1+\ldots$.

Here we get $b_{0}=1 / 2$ and $b_{n}=0$ for all $n>0$ so that $T$ terminates with the first term and $T=1 / 2$. The $W$ transformation also terminates with a single term of $1 / 2$.

Example 11: $S=1-2+4-8+16-32+\ldots$.

Here again, $T$ terminates at the first term and $T=1 / 3$. Similarly for $W$.

Example 12: $S=1+2+4+8+16+32+\ldots$.

This example indicates, in a trivial way, that the transformation may yield a value for $T$ even if $S$ is properly divergent. Here $T$ terminates and has the value -1 . $W$ behaves in the same way.

Example 13: $S=2+5 / 2+17 / 4+65 / 8+257 / 16+$ $1025 / 32+\ldots$
This is a less trivial example of a properly divergent series for which $T$ converges. Here

$T=-8+45 / 7+306 / 217+2340 / 3937+18504 / 64897$ $+147600 / 1046017+\ldots$, and converges since terms tend towards $9 / 2^{n+1}$. In fact, the general term for $T$ can be written as

$$
b_{n}=9\left[\frac{2^{n-1}}{2^{2 n-1}-1}-\frac{2^{n}}{2^{2 n+1}-1}\right]
$$

for $n>1$ and hence $T$ can be summed directly as $-8+9=1$.

This is consistent with considering $S$ as the sum of the two series:

$$
\begin{aligned}
& S_{1}=1+2+4+8+16+\ldots \\
& S_{2}=1+1 / 2+1 / 4+1 / 8+1 / 16+\ldots
\end{aligned}
$$

The sum of the first is -1 by the preceding example and the sum of the second is obviously, 2 , so that $S=S_{1}+S_{2}=1$ in agreement with the preceding.

The $W$ transformation also leads to a convergent series

$$
W=\frac{92}{11}-\frac{4131}{319}+\frac{393822}{105821}+\frac{46546812}{46521101}+\cdots
$$

This also sums to 1 . The terms tend to $27 / 2^{n+1}$ and hence this transformation does not give better results than the simpler $T$ transformation in this case.

Example 14: $S=1-2+3-4+5-6+7-8+\ldots$

This falls into the category covered by theorem 17 , since

$$
R_{n}=-\frac{n+1}{n}=-1-\frac{1}{n}
$$

Since $\alpha_{1}=-1>-2$ we have $T$ convergent. We find, in fact,

$$
T=\frac{1}{3}-\frac{2}{15}+\frac{3}{35}-\frac{4}{63}+\frac{5}{99}-\ldots
$$

whose general term may be written as

$$
b_{n}=\frac{(-1)^{n-1}}{4(2 n+1)}-\frac{(-1)^{n}}{4[2(n-1)+1]}
$$

so that it may be summed by inspection to $1 / 4$.

Hence we infer that $S=1 / 4$.

The $W$ transformation, in this case, leads to an 
even more rapidly convergent series (terms tending to $\left.1 / 4 n^{2}\right)$ :

$$
W=\frac{2}{7}-\frac{6}{119}+\frac{12}{527}-\frac{20}{1519}+\frac{30}{3479}-\cdot \cdot,
$$

which also sums to $1 / 4$.

Example 15: $S=\frac{1}{2}+\frac{B_{1}}{2}-\frac{B_{2}}{4}+\frac{B_{3}}{6}-\frac{B_{4}}{8}+\cdots$,

where the $B_{i}$ are the Bernoulli numbers:

$$
\begin{aligned}
B_{1} & =\frac{1}{6} & B_{5} & =\frac{5}{66} \\
B_{2} & =\frac{1}{30} & B_{6} & =\frac{691}{2730} \\
B_{3} & =\frac{1}{42} & B_{7} & =\frac{7}{6} \\
B_{4} & =\frac{1}{30} & B_{8} & =\frac{3617}{510}
\end{aligned}
$$

etc. This is derived from an asymptotic series and represents, by continuation, the Euler constant

$$
C=0.577 \quad 215 \quad 6649 \quad . .
$$

Putting in the numerical values for the $B_{i}$, we get $S=.5+.0833333333-.0083333333+.0039682540-$

$$
.0041666667+.0075757576-.0210927961+
$$$$
.0833333333-.4432598039+\ldots . \text {. }
$$

with a minimum term of -.00417 so that direct summation up to the minimum term may be in error several units in the third decimal. The sum is, in fact, 0.57897 at this point and is thus in error nearly 2 in the 3 rd place. If we transform the series, we get (omitting the first term as not "smoothly" related to the remaining terms, see remarks in Example 1):

$$
\begin{aligned}
T= & .5757575758+.0019305963-.0007524384+ \\
& .0005540257-.0006862537+.0013133534- \\
& .0036448151+. . . .
\end{aligned}
$$

The minimum term is only .00055 and we may thus sum to within several units in the fourth place. We may repeat the transformation to get:

$$
\begin{gathered}
T^{\prime}=.5771467498+.0001080665-.0000716028+ \\
.0000710273-.0001028454+. . \quad \text {, }
\end{gathered}
$$

with minimum term of only .000071. Repeating again, we get:
$T^{\prime \prime}=.5772117490+.0000: 71214-.0000066422 \div \ldots$,

and a last repetition gives:

$$
T^{\prime \prime \prime}=.5772154337 \text {. }
$$

This is as far as we can go with the number of terms initially taken. We may, however, consider the first terms of the successive series as an infinite sequence tending towards the desired value and thus get the equivalent series:

$$
\begin{aligned}
U= & 0.5+.0757575758+.0013891740+ \\
& .0000649992+.0000036847+. . .
\end{aligned}
$$

to which we may again apply our transformation to get:

$U^{\prime}=.5771726992+.0000422405+.0000007157+\ldots$

This may be again transformed to give

$$
U^{\prime \prime}=.5772156678
$$

which is correct to 3 in the 9 th place. If we take the sequence of sums of the $U$ series, we get a new series:

$U=.5772154337+.0000002217+.0000000124 \ldots \ldots$,

which, upon transformation, gives

$$
V^{\prime}=.5772156685
$$

which is as far as we can go with the terms taken, but which is in error by 4 in the 9 th place or somewhat poorer than $U^{\prime \prime}$. This same type of effect has been noted in applications of the method to other asymptotic series and is due to the fact that the $U$ series are not convergent but are asymptotic except that we haven't carried the calculation far enough to get to negative or increasing terms. In using the method, the direction and order of magnitude of the error made at any point is given by the first term omitted and not by the trend of those included. To show the behavior in this example more clearly, we may use an additional 4 terms in the initial series. This results in the following additional terms in the various indicated steps:

$$
\begin{aligned}
B_{9}= & 43867 / 798 ; B_{10}=174611 / 330 ; B_{11}=854513 / 138 ; \\
& B_{12}=236364091 / 2730
\end{aligned}
$$$$
\text { S: }+3.0539543303-26.4562121212+
$$$$
281.4601449275-3607.5105463980
$$

$T:+.0139641901-.0710298859+.4647849878-$ 3.8127104366

$T^{\prime}:+.0002110382-.0005961288+.0022539069-$ .0111111599 
$T^{\prime \prime}:+.0000083147-.0000139705+.0000311716-$ .0000913367

$T^{\prime \prime \prime}:+.0000004870-.0000005902+.0000008889-$ .0000017155

$T^{\text {Iv }: ~} .5772156539+.0000000313-.0000000513$

$T^{\mathrm{v}}: .5772156658$

$U:+.0000002202+.0000000119$

$U^{\prime}:+.0000000129-.0000000014$

The sign of the last term shows that $U^{\prime}$ is not a convergent series of positive terms and it is to be expected that the trend of transformed values would show a "break" corresponding to the change in sign.

We might also try the $W$ transformation on this asymptotic series. We get:

$W=.5771825397+.0000771605-.0000854277+\ldots$

which has a minimum term of similar magnitude to the second $T$ transformation.

Example 16: $S=1+1+1+1+1+1+1 \ldots$

This example illustrates the divergence of the $T$ series when $R_{n}=1$ for an infinite set of values of $n$. In this case, we get $\infty$ for the first term of $T$, the remaining terms being indeterminate if (9) is used. However, use of (5) shows all $(T)$ are infinite. If we attempt the $W$ transformation in this case, we find its terms to be indeterminate. We note that any considerations of "analytic continuation" should give $S=\lim _{x \rightarrow 1-0} 1 /(1-x) \rightarrow \infty$.

Example 17: $S=1+2+3+4+5+6+\ldots$.

This example illustrates theorem 16 since we have all $\mathrm{R}_{n}>1$ but $R_{n} \rightarrow 1$ in this case. The $T$ series is found to be

$$
T=-1-2-3-4-5-6-\ldots,
$$

which diverges, being precisely the negative of $S$. This shows a departure from what might be expected heuristically since $1+2 x+3 x^{2}+4 x^{3}+\ldots=1 /(1-x)^{2}$ is positive for $x>1$ as well as for $x<1$. The $W$ transformation gives even more unexpected results since it leads to $W=0$ in closed form, being the average between the $S$ and $\mathrm{T}$ series.

This example also illustrates that, for divergent series, we do not have the equivalent of theorems 11 or 12 , at least for $R=1$ in that one could hardly expect a "smoother" series than given here.

\section{Comparison With Known Methods}

Obviously, where use may be made of special properties, better results may be obtained than by working with a given series either directly or by application of any general numerical summing technique. For example, $\log 2$ need not be obtained from the series given in example 1 since knowledge of the properties of the logarithm permits the development of series better adapted to computing purposes. Similarly, the series used for the Euler Constant in example 14 would never be used for evaluation of the constant since far more convenient series are available. It is chiefly when a series is given whose properties are not fully known to the computer that general summing methods are of value. Even in such a case, examination of the terms may indicate a special, convenient method for summing the particular series. The transformation discussed in this paper is not to be considered as a substitute for such methods. For this reason, no comparison with the method of "summation by parts" which involves knowledge of the general expression for the $n^{\prime}$ th term and a judicious splitting of such expressions into factors or with the method of Borel, which involves recognition of integrating properties of a function by examination of a series representing it or with the method of inserting a suitable variable factor (usually successive powers of a variable) into each term of a series to obtain a function of known properties which can be evaluated under a condition corresponding to unity for each factor, will be attempted.

Of other summing methods, we will limit ourselves to the following in our comparison. In the discussion, we treat these methods as transformations of series although they are usually expressed as transformations of sequences. In most cases, moreover, we assume the original series to be prefixed by a zero term in order to get more analogous transformation.

\subsection{The Euler Transformation}

This consists in replacing the series $S=\sum_{0}^{\infty} a_{n}$ by the series

$$
E=\sum_{0}^{\infty} e_{n}
$$

where

$$
\begin{aligned}
e_{0}= & \frac{1}{2} a_{0} \\
e_{1}= & \frac{1}{2^{2}}\left(a_{0}+a_{1}\right) \\
e_{n}= & \frac{1}{2^{n+1}}\left[a_{0}+n a_{1}+\frac{n(n-1)}{2 !} a_{2}+\right. \\
& \left.\frac{n(n-1)(n-2)}{3 !} a_{3}+\ldots+n a_{n-1}+a_{n}\right] .
\end{aligned}
$$

The transformation is of particular value when the $a_{i}$ are such as to cause $e_{n}$ to vanish for $n \geq k$ since, in this case, the $E$ series terminates permitting complete summing. This special case occurs when 
$a_{n}=(-1)^{n} P(n)$ where $P(n)$ is a polynominal in $n$. It is to be noted that, in this case, $S$ is divergent with $R_{n} \rightarrow-1$ and an analytic function of $1 / n$. It is further to be noted that this transformation does not improve convergence when $S$ is a convergent series of terms of the same sign but actually leads to a new series of slower convergence than the original.

\subsection{The Hölder Transformation}

This is usually expressed as a group of transformations of various "ranks." However, transformation of rank $r$ can be considered as $r$ repetitions of transformations of first rank and we therefore restrict ourselves to the simplest case. The transformation then changes the series

$$
\begin{aligned}
S= & \sum_{0}^{\infty} a_{n} \text { into the series } H=\sum_{0}^{\infty} h_{n} \text { where } \\
h_{0}= & \frac{a_{0}}{2} \\
h_{1}= & \frac{1}{2.3}\left(a_{0}+2 a_{1}\right) \\
h_{n}= & \frac{1}{(n+1)(n+2)}\left[a_{0}+2 a_{1}+3 a_{2}+\ldots+\right. \\
& \left.n a_{n-1}+(n+1) a_{n}\right] .
\end{aligned}
$$

This transformation cannot lead to a terminating series if the original series is infinite. Like the Euler transformation, this transformation leads to a more slowly convergent series if $S$ is a convergent series all of whose terms are of like sign.

\subsection{The Cesàro Transformation}

This is also usually stated as a group of transformations of various "ranks" of complexity. The first rank is identical with the Hölder transformation. We shall take the third rank as indicative of what may be achieved with the more complex forms. The Cesàro transformation of third rank changes the series $S=\sum_{0}^{\infty} a_{n}$ into the series $C=\sum_{0}^{\infty} c_{n}$ where

$c_{0}=\frac{a_{0}}{4}$

$c_{1}=\frac{1}{4.5}\left(3 a_{0}+2 a_{1}\right)$

$$
\begin{aligned}
c_{n}= & \frac{3}{(n+1)(n+2)(n+3)(n+4)}\left[(n+2)(n+1) a_{0}+\right. \\
& (n+1) n \cdot 2 a_{1}+n(n-1) \cdot 3 a_{2}+\ldots+3 \cdot 2 n a_{n-1}+ \\
& \left.2(n+1) a_{n}\right] .
\end{aligned}
$$

This transformation also cannot lead to a terminating series if the original series is nonterminating. It also leads to a more slowly convergent series if $S$ is convergent with all terms of same sign.

\subsection{The Riesz Transformation}

This transformation also has various "ranks" of complexity. The first rank is identical with the Hölder transformation. We shall take the third rank as indicative of the more complex forms. The Reisz transformation of third rank changes the series $S=\sum_{0}^{\infty} a_{n}$ into the series $P=\sum_{n}^{\infty} p_{n}$, where

$p_{0}=\frac{a_{0}}{2^{3}}$

$p_{1}=\left(\frac{2^{3}}{3^{3}}-\frac{1}{2^{3}}\right) a_{0}+\frac{1}{3^{3}} a_{1}$

$p_{n}=\left[\frac{(n+1)^{3}}{(n+2)^{3}}-\frac{n^{3}}{(n+1)^{3}}\right] a_{0}+\left[\frac{n^{3}}{(n+2)^{3}}-\frac{(n-1)^{3}}{(n+1)^{3}}\right] a_{1}+$

$\cdots+\left[\frac{2^{3}}{(n+2)^{3}}-\frac{1}{(n+1)^{3}}\right] a_{n-1}+\frac{1}{(n+2)^{3}} a_{n}$.

This transformation, like the preceding, leads to a more slowly convergent series if $S$ is convergent with all terms of the same sign. Moreover, $P$ cannot terminate if $S$ does not do so.

\subsection{The de la Vallee Poussin Transformation}

This consists in replacing the series $S=\sum_{n}^{\infty} a_{n}$ by the series $L=\sum_{0}^{\infty} l_{n}$ where

$$
\begin{gathered}
l_{0}=\frac{a_{0}}{2} \\
l_{1}=\frac{1}{2.3}\left(a_{0}+a_{1}\right) \\
l_{n}=\frac{1}{(n+1)(n+2)}\left[a_{0}+\frac{2^{2} n}{(n+3)} a_{1}+\frac{3^{2} n(n-1)}{(n+3)(n+4)} a_{2}+\right. \\
\ldots+\frac{n^{2} \cdot n !}{(n+3)(n+4) \ldots(2 n+1)} a_{n-1}+ \\
\left.\frac{(n+1)^{2} \cdot n !}{(n+3)(n+4) \ldots(2 n+2)} a_{n}\right] .
\end{gathered}
$$

Like most of the other transformations, this transformation leads to a more slowly convergent series if $S$ is convergent with all terms of like sign. $L$ terminates only when $a_{n}$ is of the form:

$a_{n}=(-1)\left[A_{1}+n(n+2) A_{2}+(n-1) n(n+2)(n+3) A_{3}+\right.$

$$
\left.\ldots+\frac{(n+m) ! A_{m}}{(n-m+1) !(n+1)}\right]
$$

where the $A_{i}$ are constants and $m$ is finite. 


\subsection{The "Ratio" Transformation}

This consists in replacing the series $S=\sum_{0}^{\infty} a_{n}$ by the series $Z=\sum_{0}^{\infty} z_{n}$ where

$$
\begin{aligned}
& z_{n}=\frac{a_{n}}{1-R} \text { for } n<k \\
& z_{n}=\frac{a_{n}-R a_{n-k}}{1-R} \text { for } n \geq k .
\end{aligned}
$$

This transformation leads to a more rapidly convergent (more slowly divergent) series wherever $\frac{a_{n+k}}{a_{n}} \rightarrow R \neq 1$ as $n \rightarrow \infty$. We shall take $k$ as the smallest integer for which this is true. This transfor- mation leads to a terminating series when $\frac{a_{n+k}}{a_{n}}=R$ for $n$ sufficiently large.

It is again pointed out that the transformations in this paper differ from all of the above in not being linear. Moreover, except for the "Ratio" transformation, all of the above have terms dependent upon an increasing number of terms of the initial series, while the transformations considered in this paper depend upon only 3 or 4 terms of the original. This tends to make computation easier than for most of the other transformations but makes results more sensitive to "irregularities". However, results become essentially independent of which term in the original series is considered as the first.

It may be of interest to consider the various methods applied to the examples previously discussed, although this does not necessarily give a comparison of general validity.

Example 1:

$$
\begin{aligned}
S & =1-1 / 2+1 / 3-1 / 4+1 / 5-1 / 6+\ldots+\frac{(-1)^{n+1}+\ldots}{n}+\ldots \\
T & =\frac{2}{3}+\frac{1}{30}-\frac{1}{105}+\frac{1}{252}-\frac{1}{495}+\frac{1}{858}-\ldots+\frac{(-1)^{n}}{n\left(4 n^{2}-1\right)}+\ldots \\
W & =\frac{11}{16}+\frac{1}{144}-\frac{1}{576}+\frac{1}{1600}-\frac{1}{3600}+\frac{1}{7056}-\ldots+\frac{(-1)^{n+1}}{4 n^{2}(n-1)^{2}}+\ldots, \\
E & =\frac{1}{2}+\frac{1}{8}+\frac{1}{24}+\frac{1}{64}+\frac{1}{160}+\frac{1}{384}+\cdots+\frac{1}{n \cdot 2^{n}}+\ldots, \\
Z & =\frac{1}{2}+\frac{1}{4}-\frac{1}{12}+\frac{1}{24}-\frac{1}{40}+\frac{1}{60}-\cdots+\frac{(-1)^{n+1}}{2 n(n+1)}+\cdots, \\
H & =\frac{1}{2}+0+\frac{1}{12}+0+\frac{1}{30}+0+\cdots+\frac{1}{2 n(2 n-1)}+0+\cdots, \\
L & =\frac{1}{2}+\frac{1}{12}+\frac{1}{30}+\frac{1}{56}+\frac{1}{90}+\frac{1}{132}+\cdots+\frac{1}{2 n(2 n-1)}+\cdots, \\
C & =\frac{1}{4}+\frac{1}{10}+\frac{1}{15}+\frac{3}{70}+\frac{9}{280}+\frac{1}{42}+\cdots+\frac{3}{2(n+1)(n+3)}\left[1+\frac{1-(-1)^{n}}{2 n(n+2)}\right]+\cdots \\
P & =\frac{1}{8}+\frac{11}{72}+\frac{25}{288}+\frac{47}{800}+\frac{73}{1800}+\frac{107}{3528}+\cdots+\frac{1}{4 n^{2}(n+1)^{2}}\left[3\left(2 n^{2}-1\right)+(-1)^{n}\right]+\ldots
\end{aligned}
$$

Examination indicates that $W$ converges more rapidly than any of the others for the first portion of the series while $E$ is fastest for later portions, with $W$ and $T$ next and the remainder all of lower order of rapidity. The crossover point between $E$ and $T$ in size of terms is at 8 terms. However, all terms in $E$ are of the same sign while $T$ has terms alternating in sign so that the crossover for error in summing is further out at 10 terms. Corresponding crossover values for $E$ and $W$ are 13 terms and 15 terms. $Z$ is superior in convergence to the remaining 4 because its terms alternate in sign although of the same order of magnitude. It is further worth noting that $H, C$ and $P$ are irregular between even and odd terms. This same type of irregularity occurs in $L$ if no zero is prefixed to $S$ prior to transforming it. Under the same conditions, the irregularity in $H$ does not take the simple form of vanishing of even terms. The labor of computation is (not using the general form for the terms) least for $Z$, somewhat higher for $T$, higher still for $H$ and $W$, with $C$ and $P$ worst in this respect. Repetition of any transformation leads to a series of 
poorer convergence except in the case of $T, W$, and $Z$, which improve convergence again to a similar degree as the first application. For this example, therefore, there appear to be no favorable features in use of the $H, L, C$, or $P$ transformations. $E$ is best if the general form is recognized and no repetition is required. $T$ is probably best otherwise, although the higher rapidity of convergence of $W$ may justify its greater labor of computation.

Example 2:

$$
\begin{aligned}
S= & \frac{1}{2}-1+\frac{1}{4}-\frac{1}{3}+\frac{1}{6}-\frac{1}{5}+\ldots+\frac{1}{2 n}-\frac{1}{2 n-1}+\ldots, \\
T= & \frac{1}{6}-\frac{7}{15}-\frac{13}{140}-\frac{5}{63}-\frac{7}{198}-\frac{23}{715}-\ldots . \\
& \frac{8 n-3}{2 n(4 n-3)(4 n-1)}-\frac{8 n-1}{(2 n-1)\left(16 n^{2}-1\right)}-\ldots, \\
W= & -\frac{1}{8}-\frac{11}{48}-\frac{1}{12}-\frac{13}{240}-\frac{1}{30}-\frac{83}{3360}-\ldots- \\
& \frac{1}{2 n(2 n-1)}-\frac{8 n^{2}+4 n-1}{8 n(n+1)\left(4 n^{2}-1\right)}-\ldots \\
E= & \frac{1}{4}-\frac{1}{8}-\frac{5}{32}-\frac{25}{192}-\frac{19}{192}-\frac{47}{640}-\ldots, \\
Z= & \frac{1}{4}-\frac{1}{4}-\frac{3}{8}-\frac{1}{24}-\frac{1}{12}-\frac{1}{60}-\ldots-\frac{3}{4 n(2 n-3)}- \\
& \frac{1}{4 n(2 n-1)}-\ldots,
\end{aligned}
$$$$
H=\frac{1}{4}-\frac{1}{4}-\frac{1}{16}-\frac{5}{48}-\frac{1}{24}-\frac{7}{120}-\ldots \text {, }
$$$$
L=\frac{1}{4}-\frac{1}{12}-\frac{19}{240}-\frac{107}{1680}-\frac{155}{3024}-\frac{6991}{166320}-\ldots \text {, }
$$$$
C=\frac{1}{8}-\frac{1}{40}-\frac{3}{80}-\frac{73}{1680}-\frac{67}{1680}-\frac{187}{5040}-\ldots \text {, }
$$$$
P=\frac{1}{16}+\frac{7}{144}-\frac{49}{2304}-\frac{701}{19200}-\frac{877}{21600}-\frac{8167}{211680}-\ldots
$$

The general terms for $E, H, L, C$, and $R$ are not of simple form but, for large $n$, they tend to $-\frac{2}{n^{2}},-$ $\frac{\log n}{n^{2}},-\frac{\sqrt{\pi}}{2 n \sqrt{n}},-\frac{3 \log n}{n^{2}}$ and $-\frac{3 \log n}{n^{2}}$, respectively. The irregularity thus makes all the methods poorer, terms being of higher order of magnitude than in example 1, except for $Z$ which, however, has terms of constant sign in this case instead of alternating sign. None of the transformations has improved the rapidity of convergence and the last 4 have made it poorer. Repetition of any transformation leads to poorer results.

Example 3:

$$
\begin{aligned}
& S=1+0-\frac{1}{2}+0+0+\frac{1}{4}+0+0+0-\frac{1}{8}+\ldots, \\
& T=1+0-\frac{1}{2}+0+0+\frac{1}{4}+0+0+0-\frac{1}{8}+\ldots, \\
& W=1+0-\frac{1}{2}+0+0+\frac{1}{4}+0+0+0-\frac{1}{8}+\ldots, \\
& E=\frac{1}{2}+\frac{1}{4}+\frac{1}{16}-\frac{1}{32}-\frac{1}{16}-\frac{15}{256}-\frac{5}{128}-\frac{17}{1024}+\ldots,
\end{aligned}
$$

$Z$ not applicable,

$$
\begin{aligned}
& H=\frac{1}{2}+\frac{1}{6}-\frac{1}{24}-\frac{1}{40}-\frac{1}{60}+\frac{1}{42}+\frac{1}{56}+\frac{3}{224}+\frac{1}{90}+\ldots \\
& L=\frac{1}{2}+\frac{1}{6}+\frac{7}{120}+\frac{1}{56}+\frac{1}{840}-\frac{1}{176}+\ldots, \\
& C=\frac{1}{4}+\frac{3}{20}+\frac{3}{40}+\frac{11}{280}+\frac{3}{140}+\frac{5}{336}-\ldots, \\
& P=\frac{1}{8}+\frac{37}{216}+\frac{407}{3456}+\frac{211}{3200}+\frac{391}{10800}+\frac{3107}{148176}+\ldots,
\end{aligned}
$$

$T$ and $W$ obviously are identical to the original. The others are of doubtful assistance. Such a series should, of course, be handled by omitting the zero terms.

\section{Example 4:}

$$
\begin{aligned}
S & =1+\frac{1}{2}-\frac{1}{4}-\frac{1}{8}+\frac{1}{16}+\frac{1}{32}-\ldots+\frac{(-1)^{n+1}}{2^{2 n-1}}-\frac{(-1)^{n+1}}{2^{2 n}}+\ldots \\
T & =2-\frac{2}{3}-\frac{1}{3}+\frac{1}{6}+\frac{1}{12}-\frac{1}{24}-\ldots+\frac{(-1)^{n}}{3.2^{2 n-3}}-\frac{(-1)^{n+1}}{3.2^{2 n-2}}-\ldots, \\
W & =\frac{10}{7}+\frac{4}{7}-\frac{6}{7}-\frac{1}{7}+\frac{3}{14}+\frac{1}{28}-\ldots+\frac{(-1)^{n+1}}{7.2^{2 n-4}}-\frac{3(-1)^{n+1}}{7.2^{2 n-3}}+\ldots, \\
E & =\frac{1}{2}+\frac{3}{8}+\frac{7}{32}+\frac{13}{128}+\frac{17}{512}+\frac{3}{2048}-\ldots+\frac{1}{\sqrt{2}}\left(\frac{5}{16}\right)^{n / 2} \sin \left(\frac{\pi}{4}+n \arctan \frac{1}{2}\right)+\ldots,
\end{aligned}
$$




$$
\begin{aligned}
& Z=\frac{4}{5}+\frac{2}{5}=\frac{6}{5}, \\
& H=\frac{1}{2}+\frac{1}{3}+\frac{5}{48}+\frac{3}{80}+\frac{17}{480}+\frac{5}{168}+\ldots, \\
& L=\frac{1}{2}+\frac{1}{4}+\frac{11}{80}+\frac{23}{280}+\frac{151}{2880}+\frac{3133}{88704}+\ldots, \\
& C=\frac{1}{4}+\frac{1}{5}+\frac{11}{80}+\frac{53}{560}+\frac{309}{4480}+\frac{71}{1344}+\ldots, \\
& P=\frac{1}{8}+\frac{41}{216}+\frac{1145}{6912}+\frac{3921}{32000}+\frac{38129}{432000}+\frac{11135}{169344}+\ldots
\end{aligned}
$$

The general terms for the latter series are somewhat complicated but, for $n$ sufficiently large, they tend to $\frac{28}{25 n^{2}}, \frac{68}{125 n^{2}}, \frac{84}{25 n^{2}}$, and $\frac{84}{25 n^{2}}$ for $H, L, C$, and $\mathrm{P}$, respectively. The last four series are therefore definitely unsatisfactory for summing since they have larger terms than the original series and have all terms of the same sign as well. $E$ has terms of peculiar variation with somewhat slower overall convergence than the original. $T$ and $W$ are similar to the original in most respects. $Z$ is best, of course, in giving a closed form sum, and is the only one of the group that is any improvement over the original.

Example 5:

$$
\begin{aligned}
& S=\frac{1}{2}-\frac{1}{8}-\frac{1}{24}+\frac{1}{64}+\frac{1}{160}-\frac{1}{364}-\cdots+\frac{(-1)^{n}}{2 n \cdot 2^{2 n}}+\frac{(-1)^{n}}{(2 n+1) \cdot 2^{2 n+1}}+\cdots \\
& T=\frac{2}{5}-\frac{7}{80}+\frac{17}{528}+\frac{31}{2112}-\frac{49}{8160}-\frac{71}{26112}+\cdots+\frac{(-1)^{n}\left(8 n^{2}-1\right)}{n(n+1)(6 n-1) \cdot 2^{2 n+1}}+\frac{(-1)^{n-1}\left(8 n^{2}+8 n+1\right)}{(n+1)(2 n+1)(6 n+5) \cdot 2^{2 n+1}}+\cdots, \\
& W=\frac{1}{6}+\frac{9}{52}+\frac{113}{624}-\frac{533}{3200}+\frac{409}{9600}-\frac{547}{12096}-\frac{331}{96768}+\cdots+\frac{(-1)^{n-1}\left(16 n^{3}-8 n+1\right)}{n(2 n-1)(2 n-5)(7 n-1) \cdot 2^{2 n-1}}+ \\
& \frac{(-1)^{n}\left(48 n^{3}+40 n^{2}-4 n-3\right)}{n(2 n+1)(2 n-5)(7 n+6) \cdot 2^{2 n}}+\cdots, \\
& E=\frac{1}{4}+\frac{3}{32}+\frac{5}{192}+\frac{1}{1024}-\frac{29}{5120}-\frac{137}{24576}-\cdots+\frac{1}{n}\left[\left(\frac{5}{16}\right)^{n / 2} \sqrt{2} \sin \left(\frac{\pi}{4}+n \arctan \frac{1}{2}\right)-\frac{1}{2^{n}}\right]+\cdots, \\
& Z=\frac{2}{5}-\frac{1}{10}+\frac{1}{15}-\frac{1}{80}-\frac{1}{300}+\frac{1}{960}+\cdots+\frac{(-1)^{n}}{5 n(n+1) \cdot 2^{2 n+1}}-\frac{(-1)^{n}}{5(2 n+1)(2 n+3) \cdot 2^{2 n}}+\cdots, \\
& H=\frac{1}{4}+\frac{1}{24}+\frac{1}{96}+\frac{3}{320}+\frac{7}{960}+\frac{13}{2688}+\cdots, \\
& L=\frac{1}{4}+\frac{1}{16}+\frac{11}{480}+\frac{9}{896}+\frac{197}{40320}+\frac{899}{354816}+\ldots . \\
& C=\frac{1}{8}+\frac{1}{16}+\frac{17}{480}+\frac{51}{2240}+\frac{143}{8960}+\frac{127}{10752}, \\
& P=\frac{1}{16}+\frac{35}{432}+\frac{707}{13824}+\frac{407}{12800}+\frac{3673}{17200}+\frac{36023}{2370816}+\cdots
\end{aligned}
$$

The last four series have terms tending towards $\frac{1}{5 n^{2}}, \frac{2}{25 n^{2}}, \frac{3}{5 n^{2}}$, and $\frac{3}{5 n^{2}}$ respectively. $Z$ is the only transformation leading to a faster converging series. $T$ and $W$ lead to series of similar order of convergence as the originial while the remainder are of slower convergence. 
Example 6:

$$
\begin{aligned}
& S=\frac{1}{2}+\frac{1}{12}+\frac{1}{30}+\frac{1}{56}+\frac{1}{90}+\cdots+\frac{1}{2 n(2 n-1)}+\cdots, \\
& T=\frac{3}{5}+\frac{7}{180}+\frac{19}{1170}-\frac{109}{12376}+\frac{59}{10710}+\cdots+\frac{8 n^{2}-4 n-3}{2 n(2 n-1)(4 n-3)(4 n+1)}+\cdots, \\
& W=\frac{11}{16}+\frac{1}{240}+\frac{1}{1120}+\frac{1}{3360}+\cdots+\frac{3}{8 n(n-1)(2 n-1)(2 n-3)}+\cdots, \\
& Z \text { not applicable. }
\end{aligned}
$$

The remaining transformations all lead to series converging more slowly than the original since $S$ has terms all of the same sign. $T$ is not usable directly since its convergence is similar to that of
$S$ but, as indicated in a previous section, it can be combined with $S$ to obtain a more convergent series, if its trend is known. $W$ obviously converges more rapidly than $S$.

\section{Example 7:}

$$
\begin{aligned}
& S=1+\frac{1}{2}-\frac{1}{3}-\frac{1}{4}+\frac{1}{5}+\frac{1}{6}-\frac{1}{7}-\frac{1}{8}+\ldots+\frac{(-1)^{n+1}}{2 n}+\frac{(-1)^{n}}{2 n+1}+\ldots, \\
& T=2-\frac{7}{10}-\frac{17}{15}+\frac{31}{36}+\frac{49}{45}-\frac{71}{81}-\ldots+\frac{(-1)^{n}\left(8 n^{2}-1\right)}{2 n(4 n+1)}+\frac{(-1)^{n}\left(8 n^{2}+8 n+1\right)}{(2 n+1)(4 n+1)}-\ldots, \\
& W=\frac{17}{12}+\frac{1}{6}-\frac{77}{120}-\frac{1}{20}+\frac{319}{840}+\frac{1}{42}-\frac{275}{1008}-\ldots,+\frac{(-1)^{n+1}\left(16 n^{3}-12 n^{2}-2 n+1\right)}{4 n(n-1)\left(4 n^{2}-1\right)}+\frac{(-1)^{n+1}}{2 n(2 n+1)}+\cdots, \\
& E=\frac{1}{2}+\frac{3}{8}+\frac{5}{24}+\frac{5}{64}+\frac{1}{160}-\frac{7}{384}-\cdots+\frac{1}{(n+1) \cdot 2^{n+1}}\left[2.2^{n / 2} \sin n \pi / 4+1\right]+\ldots, \\
& Z=\frac{1}{2}+\frac{1}{4}+\frac{1}{3}+\frac{1}{8}-\frac{1}{15}-\frac{1}{24}+\cdots+\frac{(-1)^{n+1}}{4 n(n+1)}+\frac{(-1)^{n}}{(2 n+1)(2 n+3)}+\cdots, \\
& H=\frac{1}{2}+\frac{1}{3}+\frac{1}{12}+0+\frac{1}{30}+\frac{1}{21}+\cdots+\frac{1}{2(2 n+1)(4 n+1)}+\frac{1}{(2 n+1)(4 n+3)}+\frac{1}{4(n+1)(4 n+3)}+0+\cdots, \\
& L=\frac{1}{2}+\frac{1}{4}+\frac{2}{15}+\frac{3}{40}+\frac{2}{45}+\frac{1}{36}+\cdots \text {, } \\
& C=\frac{1}{4}+\frac{1}{5}+\frac{2}{15}+\frac{3}{35}+\frac{17}{280}+\frac{1}{21}+\cdots+\frac{3}{\left(4 n^{2}-1\right)}+\frac{3}{4 n(n+1)}\left[1+\frac{(-1)^{n}}{4 n^{2}-1}\right]+\cdots, \\
& P=\frac{1}{8}+\frac{41}{216}+\frac{71}{432}+\frac{47}{400}+\frac{433}{5400}+\frac{4325}{74088}+\cdots,
\end{aligned}
$$

The $L$ and $P$ series have terms of the order of $1 / n^{2}$ for large $n$. For this series, therefore, $E$ gives the most rapid convergence, although with terms of peculiar variation, while $T$ diverges. $Z$ is more rapidly convergent than $S$. All remaining methods give terms of similar order of convergence to that of the original series. $Z$ is thus preferable to all those listed for ease of summing and is, in addition, much simpler to evaluate.
Examples 8 and 9. These were inserted for theoretical interest to show that the $T$ and $W$ transformations were not related in the sense that the field of application of either included that of the other. In neither example is the $Z$ transformation applicable and, in both examples, all the other transformations considered lead to series of slower convergence since terms are all of the same sign. 
Example 10:

$$
\begin{aligned}
S= & 1-1+1-1+1-1+1-1+\ldots \\
T= & \frac{1}{2} \\
W= & \frac{1}{2} \\
E= & \frac{1}{2} \\
Z= & \frac{1}{2} \\
H= & \frac{1}{2}-\frac{1}{6}+\frac{1}{6}-\frac{1}{10}+\frac{1}{10}-\frac{1}{14}+\frac{1}{14}-\cdots \cdot \\
& \frac{1}{2(2 n+1)}+\frac{1}{2(2 n+1)}+\cdots \\
L= & \frac{1}{2} \\
C= & \frac{1}{4}+\frac{1}{20}+\frac{1}{20}+\frac{3}{140}+\frac{3}{140}+\frac{1}{84}+\cdots+ \\
& \frac{3}{4\left(4 n^{2}-1\right)}+\frac{3}{4\left(4 n^{2}-1\right)}+\cdots \cdot \\
P= & \frac{1}{8}+\frac{29}{216}+\frac{23}{432}+\frac{79}{2000}+\frac{23}{1000}+\frac{153}{8232}+\cdots+ \\
& \frac{12 n^{2}+14 n+3}{8 n(2+1)^{3}}+\frac{12 n^{2}+10 n+1}{8(n+1)(2 n+1)^{3}}+\cdots \cdot
\end{aligned}
$$

For this example, $T, W, E, Z$, and $L$ all lead to series terminating with a single term. $H, C$, and $P$ lead to convergent series. Although the terms in $H$ are of higher order of magnitude, they are of alternating sign and trivial to sum. Since $\frac{1}{2 n+1}-$ $\frac{1}{2 n+3}=\frac{2}{(2 n+1)(2 n+3)}: C$ is readily summed to a total of $\frac{1}{2}$. Also, we have

$$
\begin{aligned}
& \frac{12 n^{2}+14 n+3}{8 n(2 n+1)^{3}}+\frac{12 n^{2}+10 n+1}{8(n+1)(2 n+1)^{3}} \\
& =\frac{3}{8 n(n+1)}=\frac{3}{8}\left(\frac{1}{n}-\frac{1}{n+1}\right)
\end{aligned}
$$

so that $P$ is readily summed to $\frac{1}{8}+\frac{3}{8}=\frac{1}{2}$. We thus see that all methods are consistent with each other in this example but that $H, C$, and $P$ are most difficult to apply and evaluate.
Example 11:

$$
\begin{aligned}
S & =1-2+4-8+16+32+\ldots+(-2)^{n}+\ldots, \\
T & =\frac{1}{3}, \\
W & =\frac{1}{3}, \\
E & =\frac{1}{2}-\frac{1}{4}+\frac{1}{8}-\frac{1}{16}+\frac{1}{32}-\frac{1}{64}+\ldots+\frac{(-1)^{n}}{2^{n+1}}+\ldots, \\
Z & =\frac{1}{3}, \\
H & =\frac{1}{2}-\frac{1}{2}+\frac{3}{4}-\frac{23}{20}+\frac{19}{20}-\frac{45}{14}+\ldots, \\
L & =\frac{1}{2}-\frac{1}{6}+\frac{1}{60}-\frac{1}{140}-\frac{1}{630}-\frac{1}{693}-\ldots, \\
C & =\frac{1}{4}-\frac{1}{20}+\frac{1}{10}-\frac{1}{14}+\frac{31}{280}-\frac{1}{8}+\ldots, \\
P & =\frac{1}{8}+\frac{7}{72}+\frac{7}{576}+\frac{301}{8000}-\frac{73}{9000}+\frac{827}{24696}-\cdots,
\end{aligned}
$$

For this case, $T, W$, and $Z$ lead to terminating series while $E$ and $L$ lead to convergent series (terms for the latter tend to $\left.\frac{9(-1)^{n} \sqrt{\pi n}}{2 \cdot 8^{n+1}}\right)$ with $H, C$, and $P$ not of sufficient power to lead to series that converge. Furthermore, if a similar series of larger modulus were taken, say

$$
\begin{aligned}
S^{\prime}= & 1-6+36-216+1296-7776+\ldots+ \\
& (-6)^{n}+\ldots
\end{aligned}
$$

we would have $E$ and $L$ diverge as well with

$$
\begin{aligned}
E^{\prime}= & \frac{1}{2}-\frac{5}{4}+\frac{25}{8}-\frac{125}{16}+\frac{625}{32}-\frac{3125}{64}+\ldots+ \\
& \frac{(-1)^{n}}{2}\left(\frac{5}{2}\right)^{n}+\ldots
\end{aligned}
$$

and

$$
L^{\prime}=\frac{1}{2}-\frac{5}{6}+\frac{13}{12}-\frac{37}{28}+\frac{7}{6}-\ldots
$$

with terms tending to $\frac{3(-1)^{n} \sqrt{\pi n}}{2}\left(\frac{25}{24}\right)^{n}$. However, in this case, $E$ and $L$ could be repeated to obtain equivalent convergent series. It is easy to sum $E$ as given since it is a geometric series. It is found to agree with the single term values obtained for $T, W$, and $Z$. It can be shown that $L$ also sums to $1 / 3$. 
Example 12:

$$
\begin{aligned}
S & =1+2+4+8+16+\ldots+2^{n}+\ldots, \\
T & =-1, \\
W & =-1, \\
Z & =-1 .
\end{aligned}
$$

All other transformations lead to properly divergent series. Thus $T, W$, and $Z$ are the only methods that assign meaning to this type of properly divergent series. They agree in value assigned.

\section{Example 13:}

$$
\begin{aligned}
S= & 2+\frac{5}{2}+\frac{17}{4}+\frac{65}{8}+\frac{257}{16}+\frac{1025}{32}+\ldots+\frac{2^{2 n}+1}{2^{n}}+\ldots, \\
T= & -8+\frac{45}{7}+\frac{306}{217}+\frac{2340}{3937}+\frac{18504}{64897}+\ldots . \\
& \frac{9 \cdot 2^{n-1}\left(2^{2 n}+1\right)}{\left(2^{2 n-1}-1\right)\left(2^{2 n+1}-1\right)}+\ldots
\end{aligned}
$$$$
W=\frac{92}{11}-\frac{4131}{319}+\frac{396822}{105821}+\frac{46546812}{46521101}+\ldots+
$$$$
\frac{27 \cdot 2^{n-2}\left(2^{2 n-2}+1\right)\left(2^{2 n-1}+1\right)\left(2^{2 n}+1\right)}{\left(2^{4 n-4}-7 \cdot 2^{2 n-2}+1\right)\left(2^{4 n}-7 \cdot 2^{2 n}+1\right)}+\ldots .
$$$$
Z=-2+\frac{3}{2}+\frac{3}{4}+\frac{3}{8}+\frac{3}{16}+\frac{3}{32}+\frac{3}{64}+\ldots+\frac{3}{2^{n}}+\ldots
$$

As for the preceding example, all other transformations lead to properly divergent series. $Z$ is easily summed to give $-2+3=+1$ in agreement with the sum of the $T$ or $W$ series. $Z$ is, of course, simpler to apply and sum than is $T$ and either is simpler than $W$.

Example 14:

$$
\begin{aligned}
S= & 1-2+3-4+5-6+\ldots-(-1)^{n} n+\ldots \\
T= & \frac{1}{3}-\frac{2}{15}+\frac{3}{35}-\frac{4}{63}+\frac{5}{99}-\ldots-\frac{(-1)^{n} n}{4 n^{2}-1}+\ldots, \\
W= & \frac{2}{7}-\frac{6}{119}+\frac{12}{527}-\frac{20}{1519}+\frac{30}{3479}-\ldots . \\
& \frac{(-1)^{n} n(n+1)}{\left(2 n^{2}-1\right)\left(2 n^{2}+4 n+1\right)}-\ldots, \\
E= & \frac{1}{2}-\frac{1}{4}=\frac{1}{4} \\
Z= & \frac{1}{2}-\frac{1}{2}+\frac{1}{2}-\frac{1}{2}+\frac{1}{2}-\frac{1}{2}+\ldots \\
H= & \frac{1}{2}-\frac{1}{2}+\frac{1}{2}-\frac{1}{2}+\frac{1}{2}-\frac{1}{2}+\ldots
\end{aligned}
$$

$$
\begin{aligned}
L= & \frac{1}{2}-\frac{1}{6}-\frac{1}{30}-\frac{1}{70}-\frac{1}{126}-\cdots-\frac{1}{2\left(4 n^{2}-1\right)}-\cdots, \\
C= & \frac{1}{4}-\frac{1}{20}+\frac{1}{20}-\frac{3}{140}+\frac{3}{140}-\frac{1}{84}+\cdots \cdot \\
& \frac{3}{4\left(4 n^{2}-1\right)}+\frac{3}{4\left(4 n^{2}-1\right)}-\cdots, \\
P= & \frac{1}{8}+\frac{7}{72}-\frac{1}{288}+\frac{17}{800}-\frac{7}{1800}+\cdots+ \\
& \frac{2 n^{2}+4 n+1}{8 n^{2}(2 n+1)^{2}}-\frac{2 n^{2}-1}{8(n+1)^{2}(2 n+1)^{2}}+\cdots
\end{aligned}
$$

Now we have the identities:

$$
\begin{gathered}
\frac{n}{4 n^{2}-1}=\frac{1}{4(2 n-1)}+\frac{1}{4(2 n+1)} \\
\frac{n(n+1)}{\left(2 n^{2}-1\right)\left(2 n^{2}+4 n+1\right)}=\frac{1}{4\left(2 n^{2}-1\right)}+\frac{1}{4\left(2 n^{2}+4 n+1\right)} \\
\frac{1}{2\left(4 n^{2}-1\right)}=\frac{1}{4(2 n-1)}-\frac{1}{4(2 n+1)} \\
\frac{2 n^{2}+4 n+1}{8 n^{2}(2 n+1)^{2}-\frac{2 n^{2}-1}{8(n+1)^{2}(2 n+1)^{2}}} \\
=\frac{2 n+1}{8 n^{2}(n+1)^{2}}=\frac{1}{8 n^{2}}-\frac{1}{8(n+1)^{2}}
\end{gathered}
$$

Hence we may readily sum all of the convergent series above, obtaining $\frac{1}{4}$ in all cases. Also, from example 10 , the $Z$ and $H$ series may be summed by repetition or otherwise to this same sum. All the given methods are therefore consistent for this example. $E$ is best since it terminates. $T$ is not quite as good as $W, C$, or $P$ in rate of convergence.

Example 15:

$$
\begin{aligned}
S= & \frac{1}{2}+\frac{1}{12}-\frac{1}{120}+\frac{1}{252}-\frac{1}{240}+\frac{1}{132}-\frac{691}{32760}+ \\
& \frac{1}{12} \cdots-\frac{(-1)^{n} B n}{2 n}+\cdots, \\
T= & \frac{19}{33}+\frac{79}{40920}-\frac{241}{320292}+\frac{169}{305040}-\frac{29011}{42274452}+\cdots, \\
W= & \frac{2909}{5040}+\frac{1}{12960}-\frac{31}{362880}+\cdots, \\
E= & \frac{13}{24}+\frac{3}{160}+\frac{89}{10080}+\frac{37}{8960}+\frac{299}{147840}+\frac{323}{366080}+\cdots, \\
Z= & \text { not applicable, }
\end{aligned}
$$


$H=\frac{13}{24}+\frac{1}{90}+\frac{11}{1680}+\frac{13}{4200}+\frac{461}{138600}-\frac{67}{105105}+\cdots$,

$L=\frac{13}{24}+\frac{1}{80}+\frac{19}{3150}+\frac{83}{23520}+\frac{6759}{2910600}+\frac{18175}{11099088}+\cdots$,

$C=\frac{25}{48}+\frac{7}{600}+\frac{97}{12600}+\frac{79}{14700}+\frac{5279}{1293600}+\frac{45229}{15135120}+\cdots$,

$P=\frac{49}{96}+\frac{181}{12960}+\frac{1777}{181440}+\frac{387}{56000}+\frac{125879}{24948000}+\cdots$

In applying all transformations, the first term in $S$ was omitted during transformation and then added to the first term of the result since it is not smoothly related to the following terms. Terms far out in each series tend to have values $-\frac{(-1)^{n} \pi^{2} B_{n}}{n^{4}},+$ $\frac{3(-1)^{n} \pi^{4} B_{n}}{n^{7}},-\frac{(-1)^{n} B_{n}}{2 n \cdot 2^{n}},-\frac{(-1)^{n} B_{n}}{2 n^{2}},-\frac{\sqrt{\pi}(-1)^{n} B_{n}}{8 n \cdot 2^{2 n}}$, $-\frac{3(-1)^{n} B_{n}}{n^{4}}$ and $-\frac{(-1)^{n} B_{n}}{2 n^{4}}$ for $T, W, E, H, L, C$, and $P$ respectively. Hence all the series diverge. However, these values are not approximated during the early portions of the series which are the most important for summing. In these early portions, given above, $W$ appears best, while $T$ is convenient because it is easier to compute than most of the others. All the transformations can be repeated on the later portions of the series to obtain, with suitable manipulation, any desired accuracy of evaluation. However, only $T$ and $W$ have earlier terms fitting smoothly with later terms as concerns sign.

Examples 16 and 17: These were included in earlier discussion to show the behavior of the $T$ and $W$ transformations when $R=1$. The $Z$ transformation is not applicable in either case, while all the other transformations considered lead to properly divergent series since $S$ is of that character.

The author is greatly indebted to Alexander M. Ostrowski, Otto Szasz, and Franz L. Alt for pointing out several errors in the first draft of this paper. He is particularly indebted to Olga Taussky, who carefully reviewed parts of the final manuscript and whose suggestions led to material improvement of the paper. Ida Rhodes also contributed substantially by checking the numerical work.

Washington, November 2, 1950. 